$$
\text { ص ص[1-1-10] }
$$

العلاقة بين نشاط الاستثمار في الأوراق المالية والنمو الاقتصادي

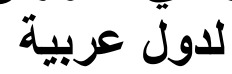

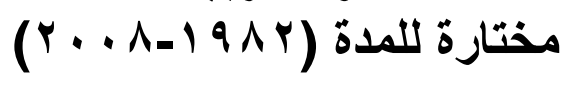

$$
\text { مدرس - المعهد التقني عمر }
$$

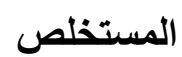

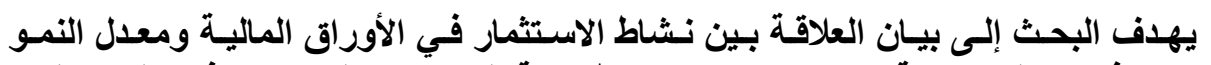

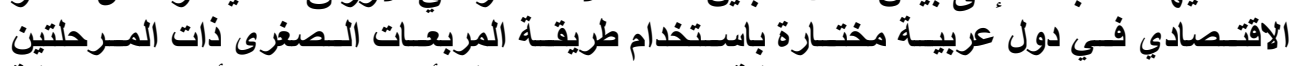
(2.S.L.S)

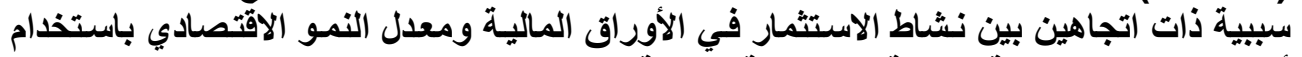

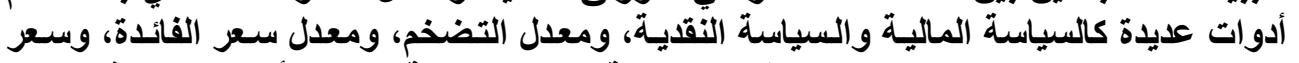

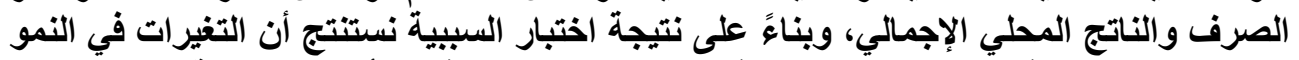

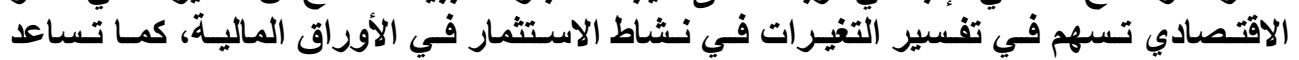

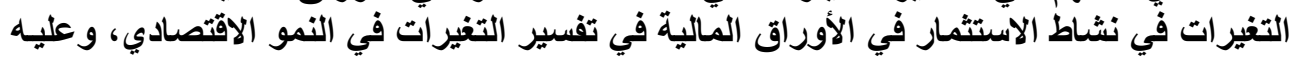

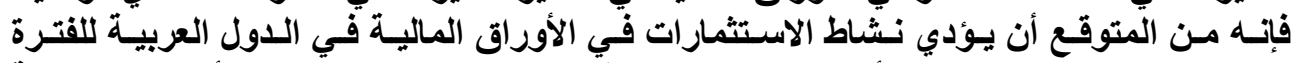

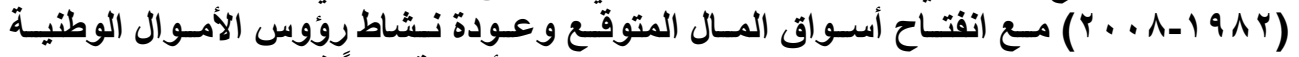

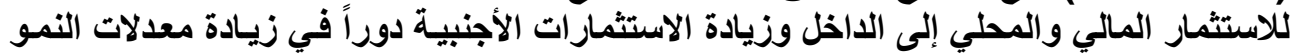
الاقتصادي.

الكلمات المفتاحية: نشاط الاستثمار في الأوراق المالية، النمو الاقتصادي. 


\title{
Relation between Investment Activity in Capitals Market and Economic Growth Rate in Selection Arabic Countries (1982-2008)
}

\author{
Dina A. Omar \\ Lecturer \\ Ninavah Technical Institution \\ Dina_amor333@yahoo.com
}

\begin{abstract}
The research aims to explain the relationship between investment activity in capitals market and economic growth rate in selected Arab countries. This was done by using method of least squares two stages to test and determine the direction of the relationship between two variables. The effects have shown that causative relationship of directions between investment activity in capitals market and economic growth by using many tools as financial and monetary policies, inflation rate, interest rate, exchange rate, gross domestic product, according to effect testing. The causative one concludes that changes in economic growth may participate to explain the investment activity of capitals markets. It also changes the investment activity in capitals market and participates to explain the economic growth. For this reason, it is expected that investment activity in capitals market in Arab countries (1982 - 2008) with opening the expected capitals market and local capitals. The resumption of expected capitals market, reactivity of local capitals to invest financially inside, increasing the foreign investments have the essential role in economic growth.
\end{abstract}

Keys words: investment activity in capitals Market, Economic Growth rate.

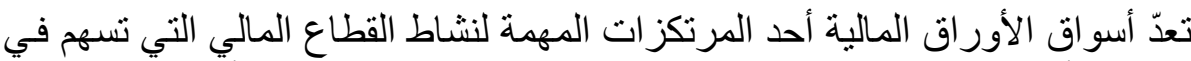

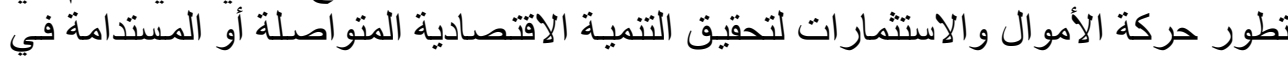

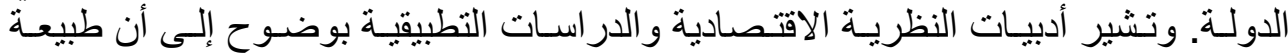

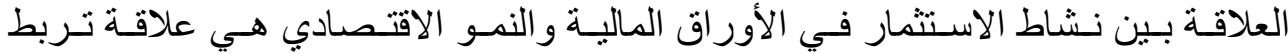

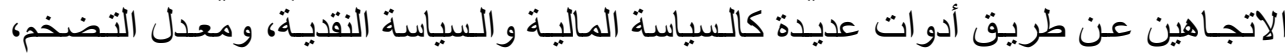

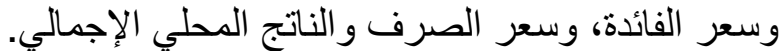

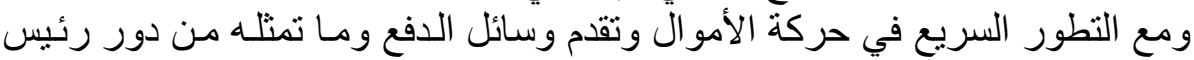

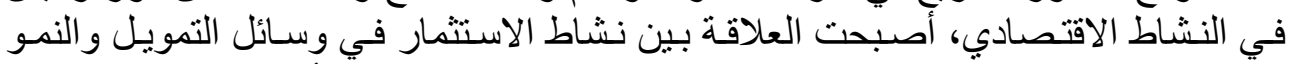

الاقتصادي محور اهتمام الكتاب والباحثين في كثثر من الدراسات والأبحاث الاقتصادية في في فئي

العقدين الأخيرين سواء في الجانب الني النظري أو التطبيقي.

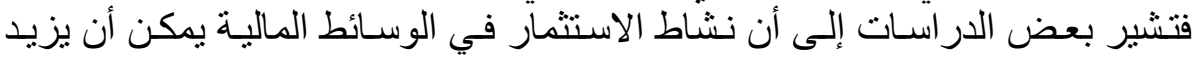

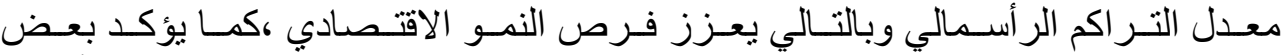

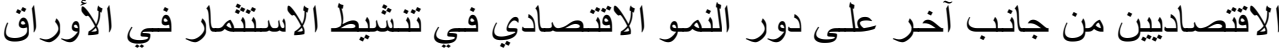

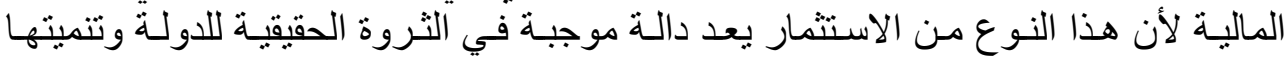

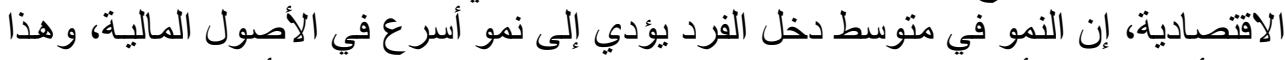

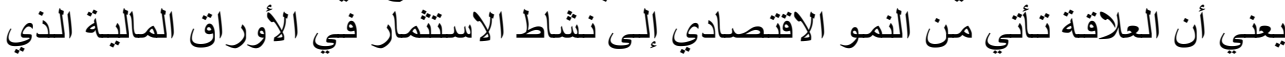




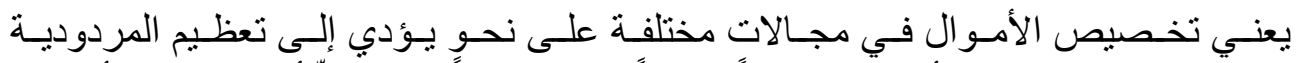

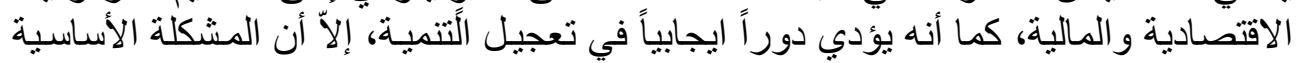

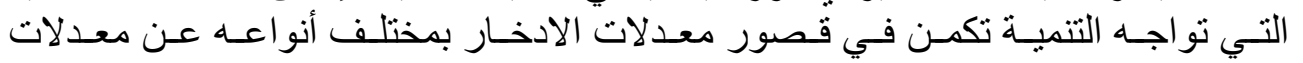

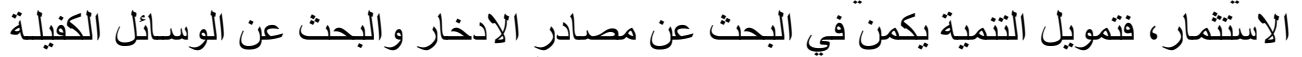

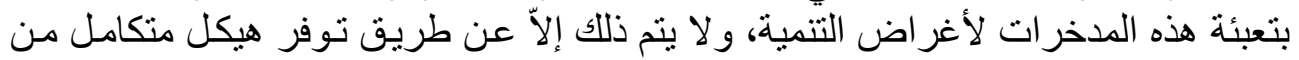

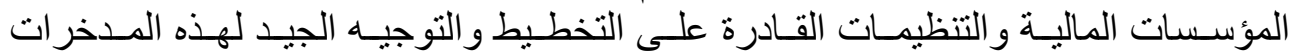

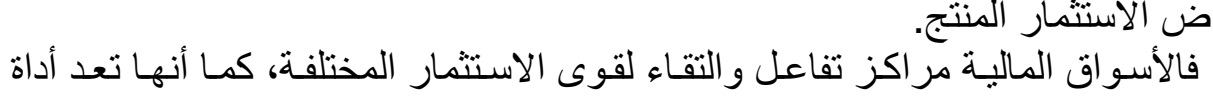

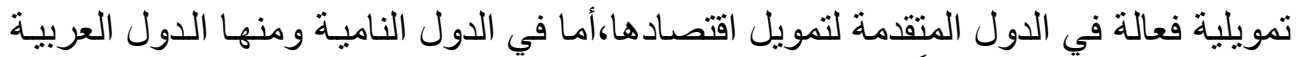

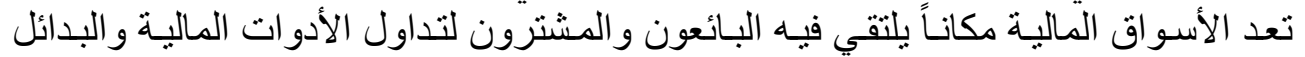

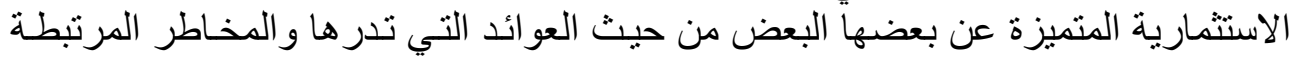

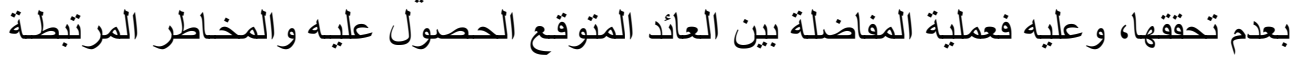

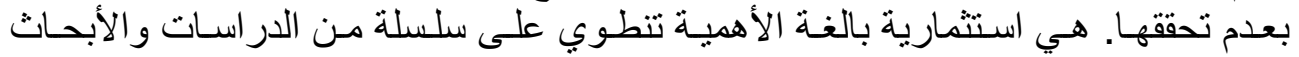

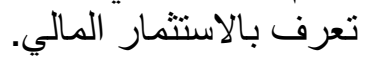

تتمثل في الإجابة على السؤال الآتي: هل أن نشاط الاستثمار في الأوراق المالية في مشكلة البحث

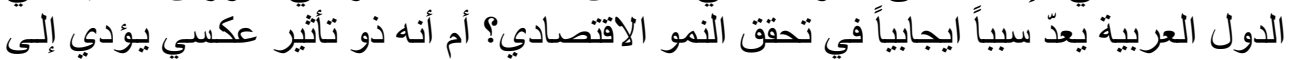

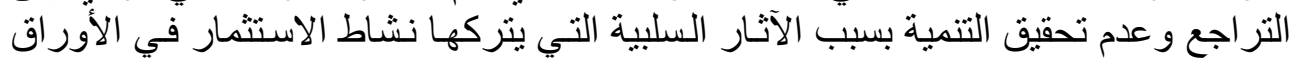

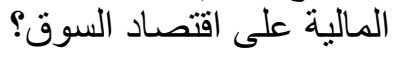

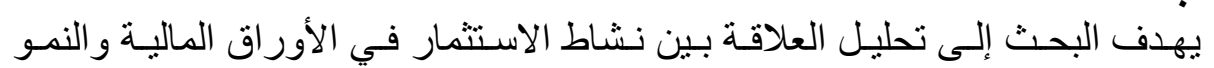

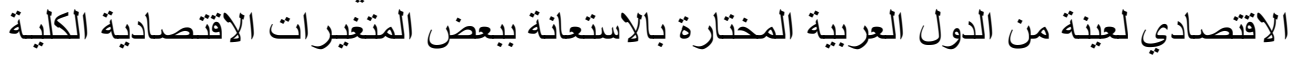

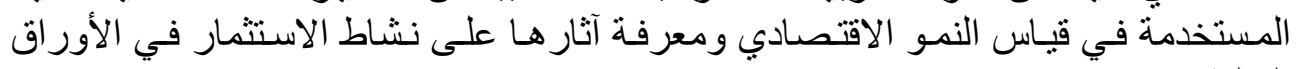

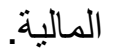

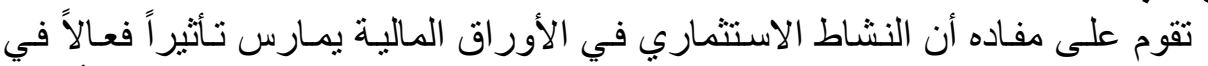

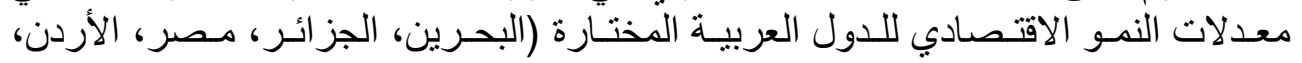

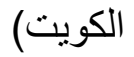

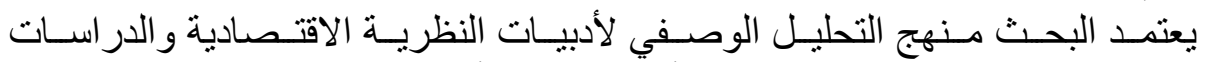
منهجية البحث

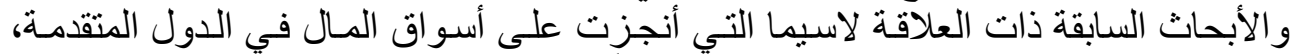

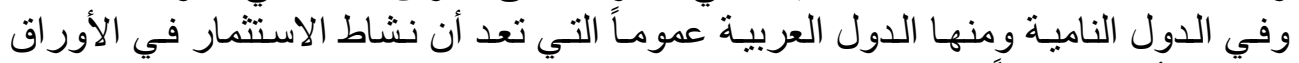

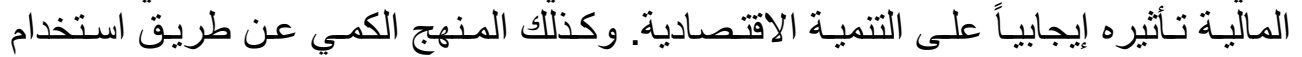

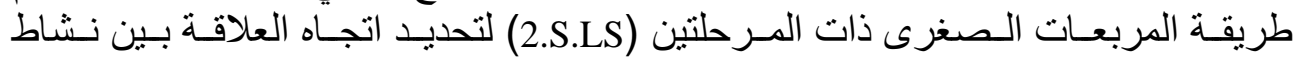


الاستثمار في الأوراق المالية ومعدلات النمو الاقتصادي لعينة من الدول العربيـة المختارة

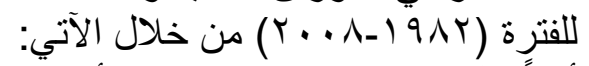

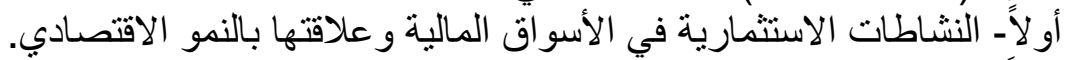

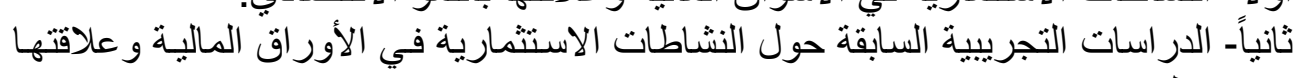
بالنمو الاقتصادي.

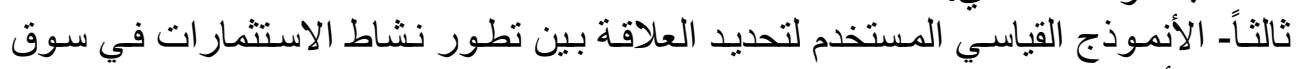
الأوراق المالية والنمو الاقتصادي لدول الفيدم عربية مختارة.

أولاًـ- النشاطات الاستثمارية في الأسواق المالية وعلاقتها بالنمو الاقتصادي

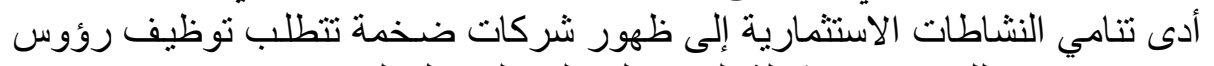

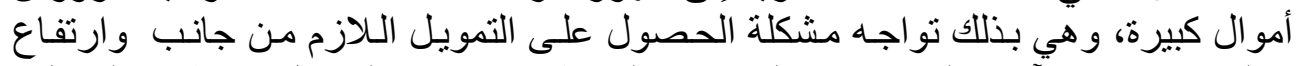

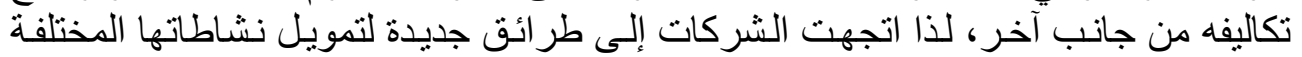

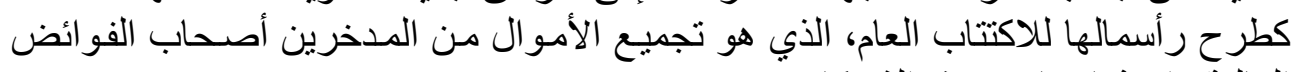

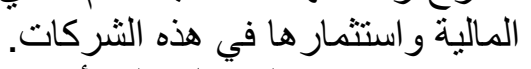

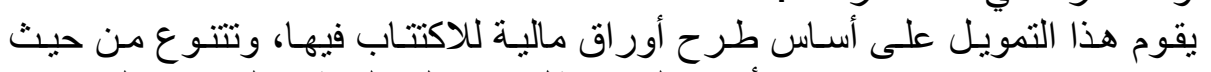

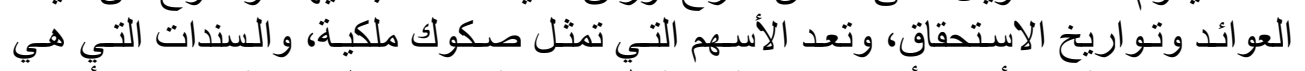

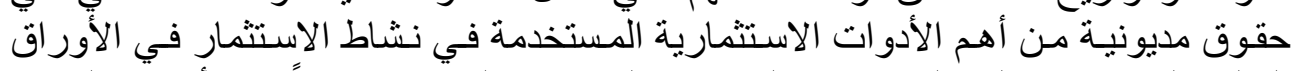

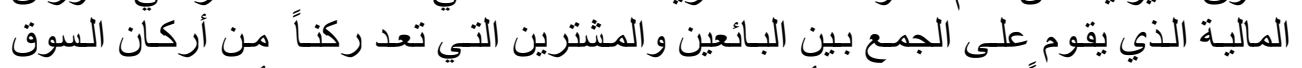

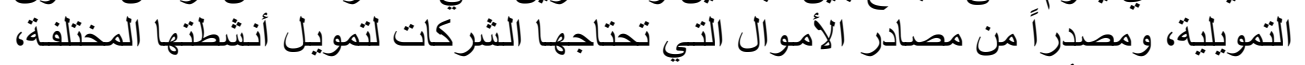

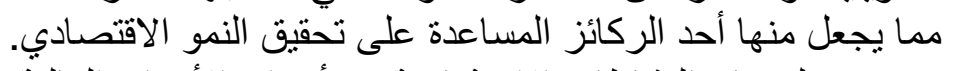

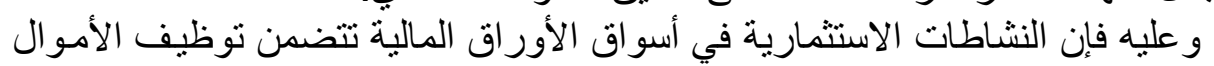

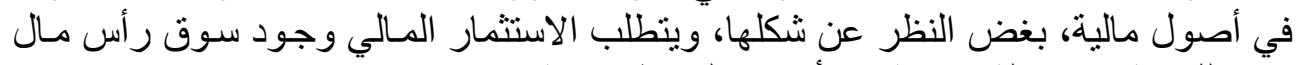

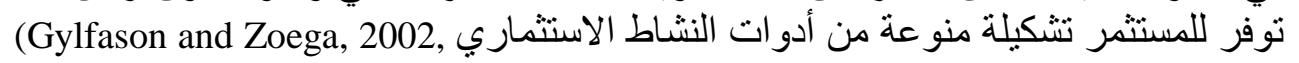

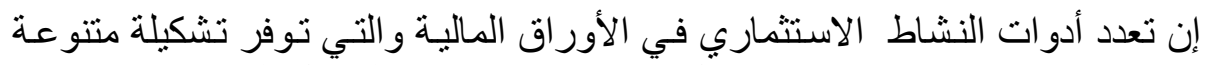

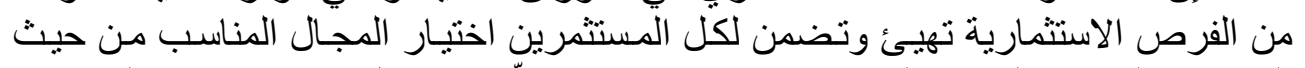

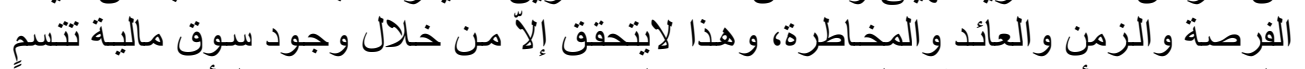

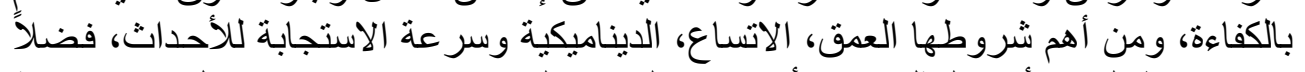

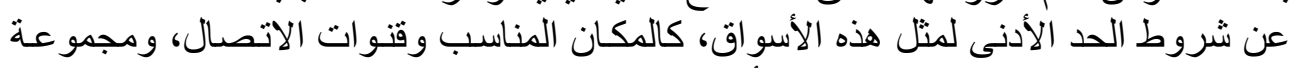

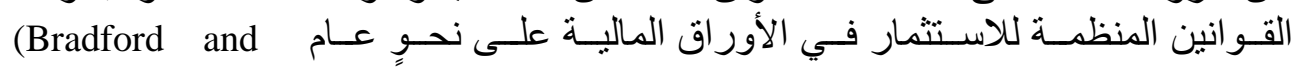
.Long,1990,40)

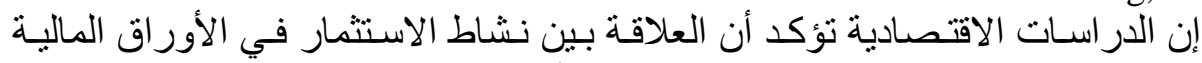

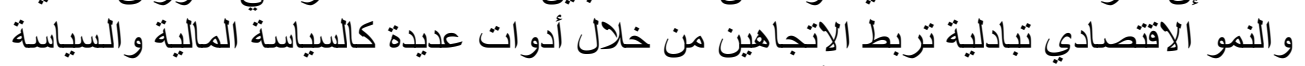

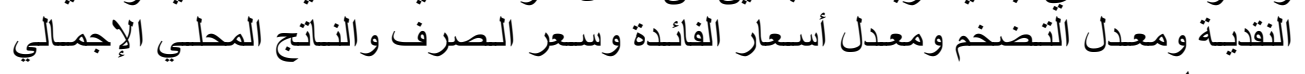
وغير ها.

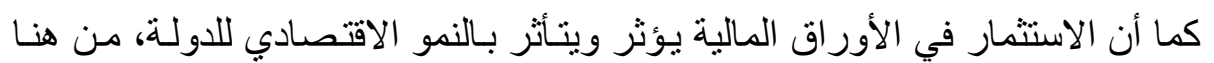

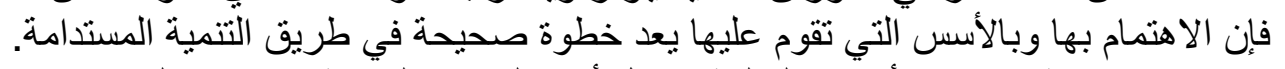

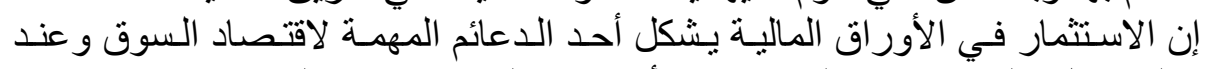

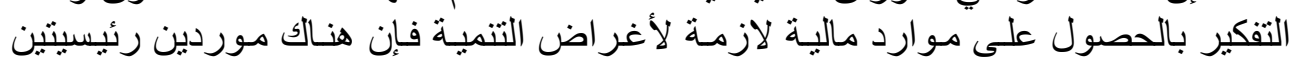




\section{[^ه]}

لتحقيق ذلك، هما المورد الداخلي والمورد الخارجي و المصدر ان لهما دور مهم وحيوي في الإي

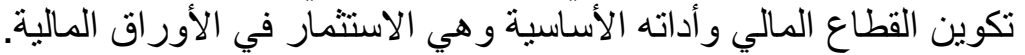

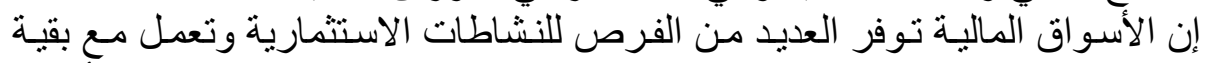

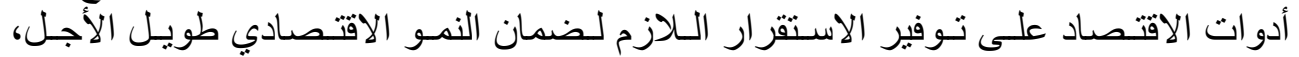

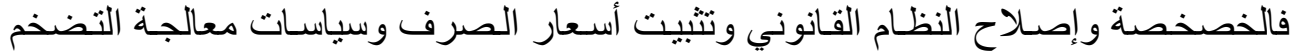

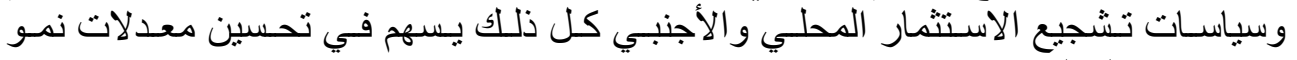
اقتصناديات الدولة.

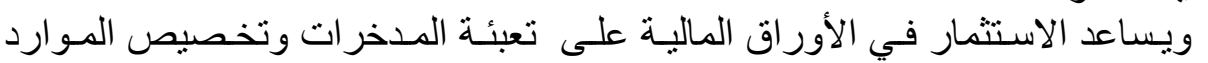

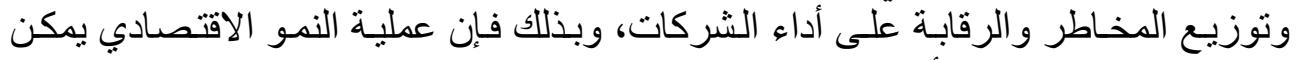

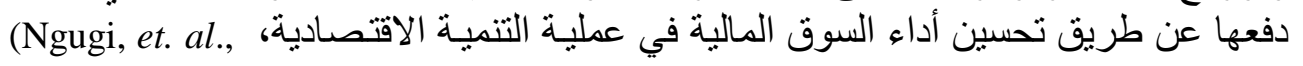
2004, 18)

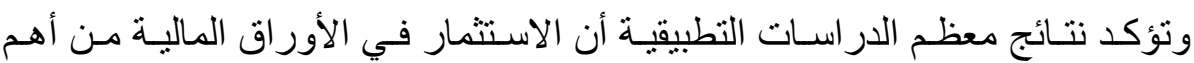

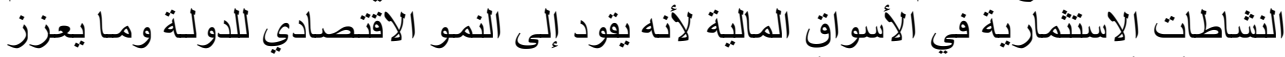

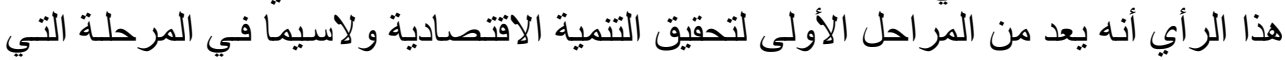

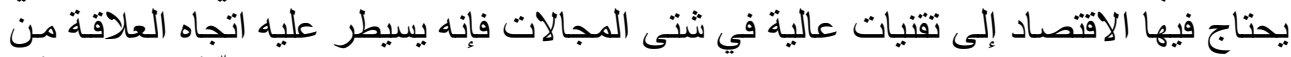

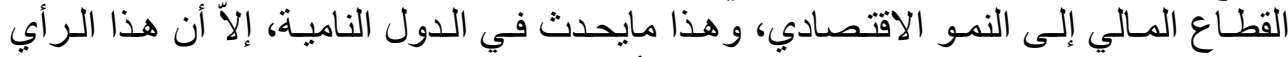

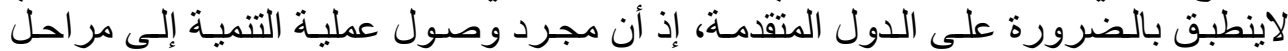

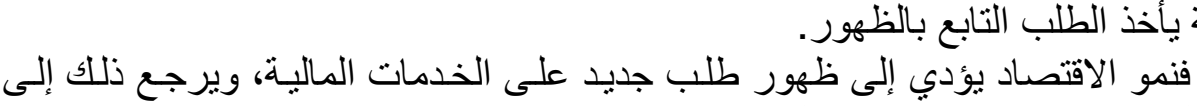

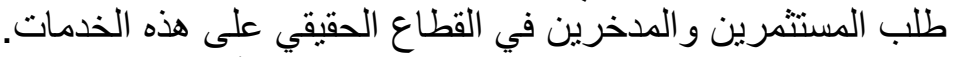
وفي هذه الحالة فإن نشاط الاستثمار في الأوراق الماليـة يعدّ عمليـة مستمرة ويسهر في تحقيق النمو الاقتصادي.

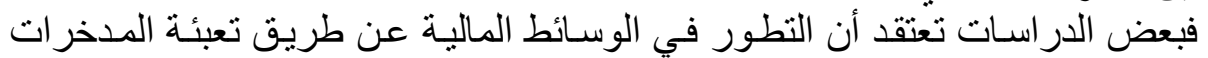

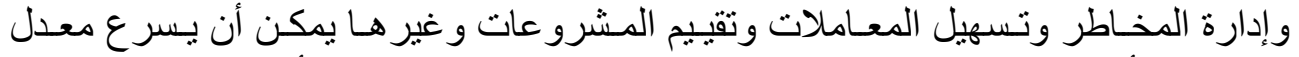

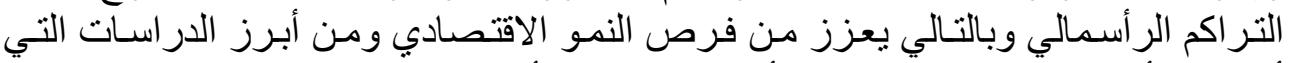

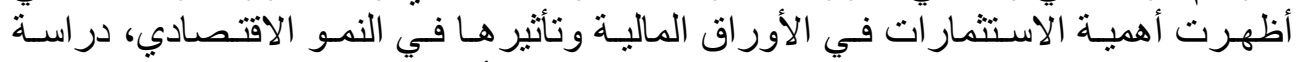

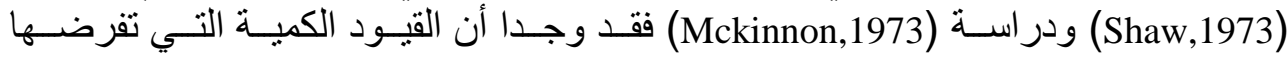

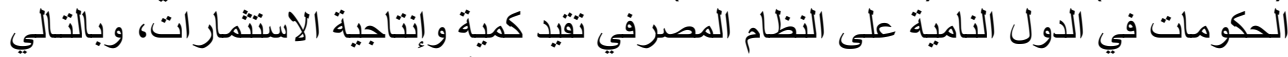

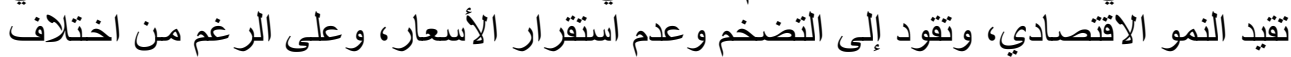

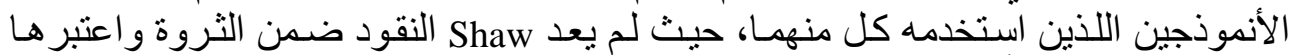

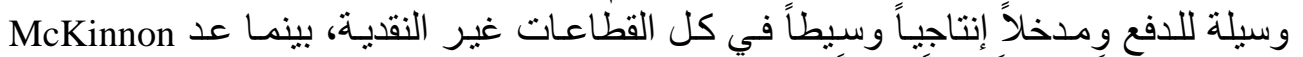

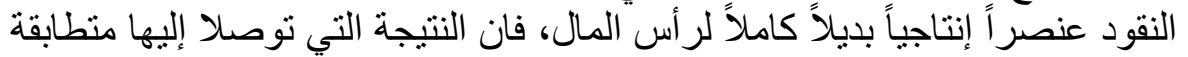

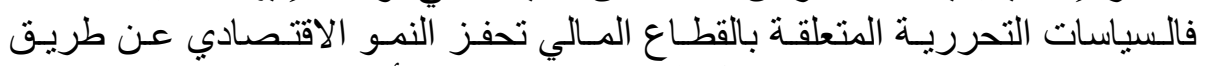

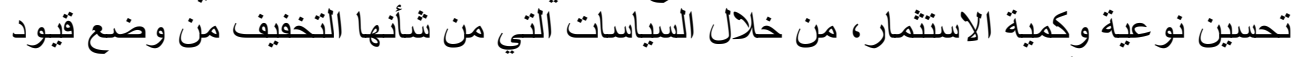

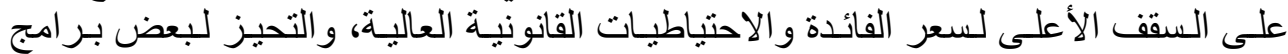

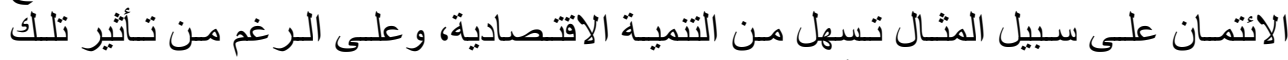

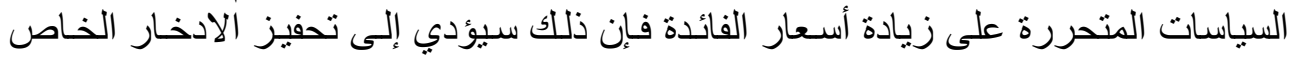

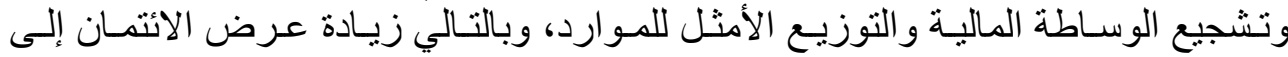
القطاع الخاص الذي بدوره يؤدي إلى زيادة الاستثمار وارتفاع الألى معدل النمو الاقتصادي. 


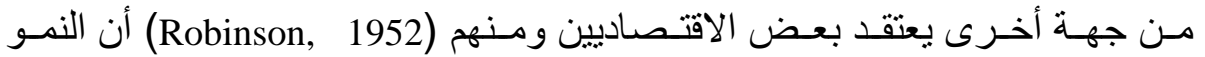

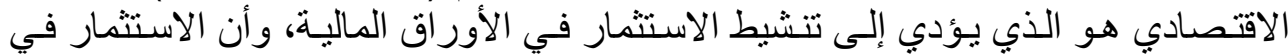

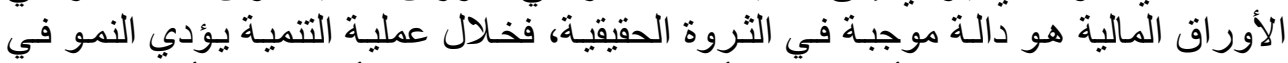

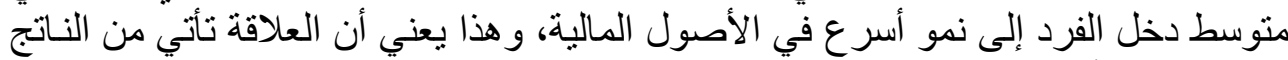
القومي إلى الأصول المبرد المالية.

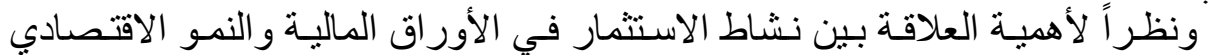

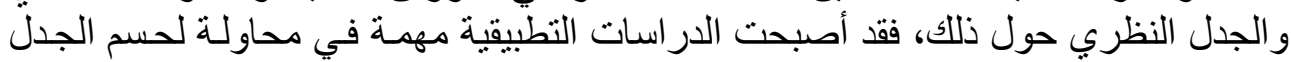

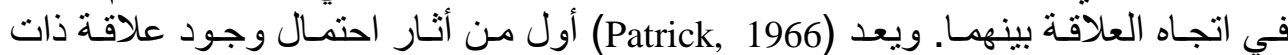

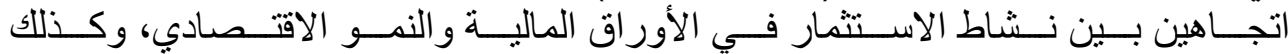
(Goldsmith,1969)

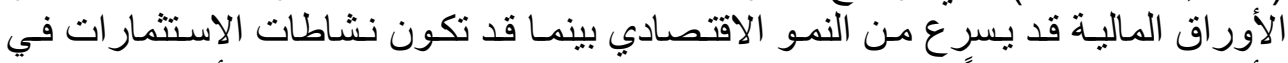

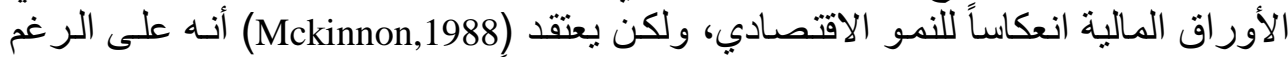

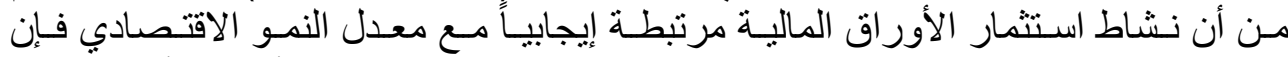

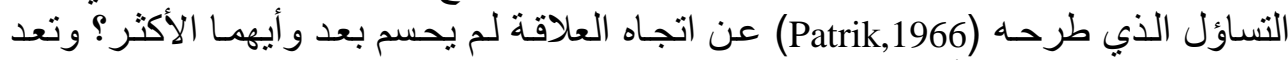

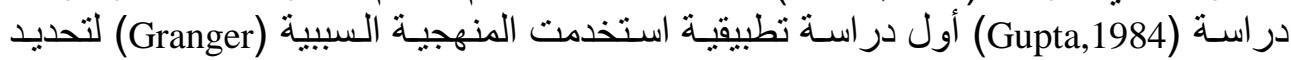
العلاقة السببية بين نشاط الاستثمار في الأوراق المالية والنمو الاقتصادي (القدير، ع ـ. ب.،

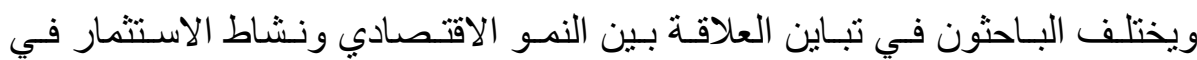

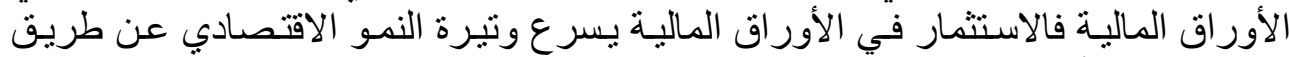

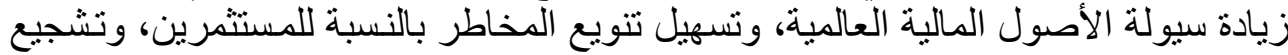
القرار ات الاستثمارية الواعية بناء على المعلومات الماتية المتاحة، وزيادة الإنتاجية من خلادل حثل

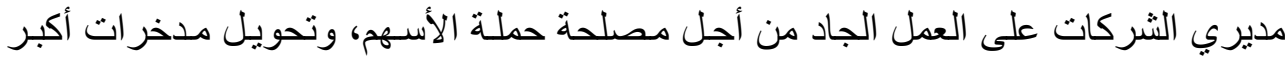

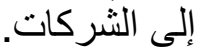

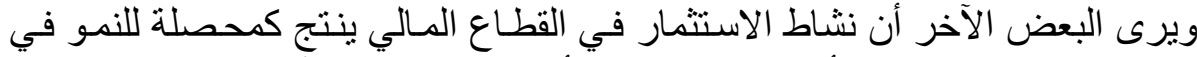

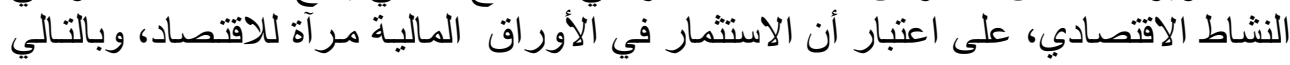

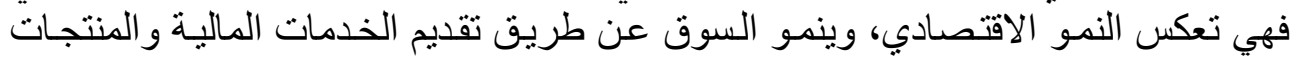
الاستثمارية اللازمة للتطور الاقتصادي (Vazakidis and Adamopoulos, 2010,9).

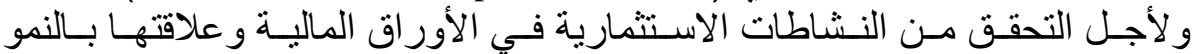

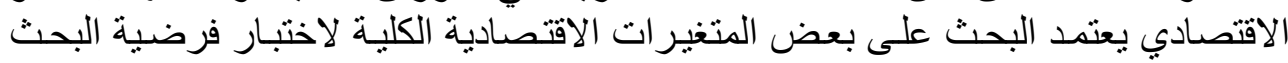

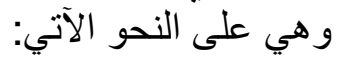

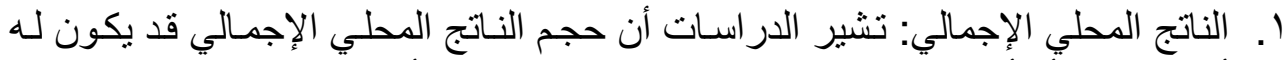

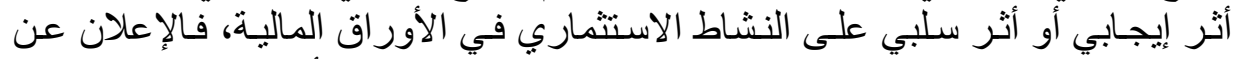

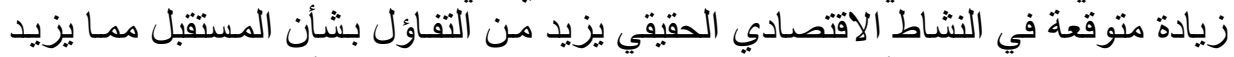

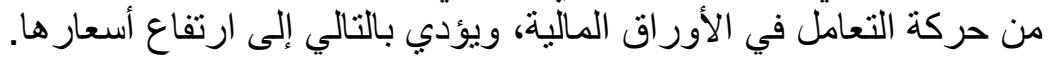

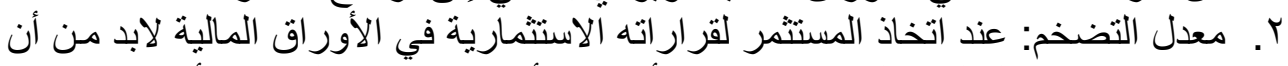

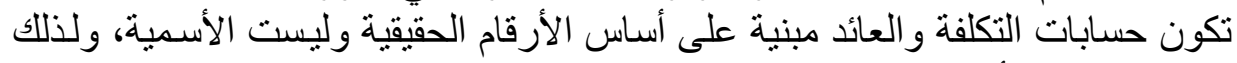

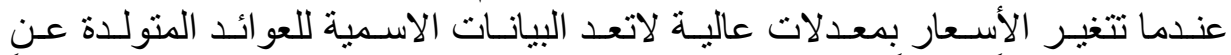

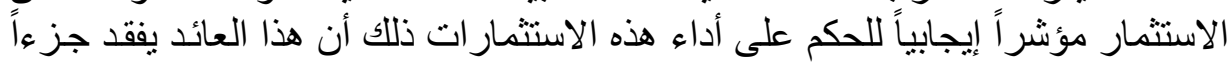




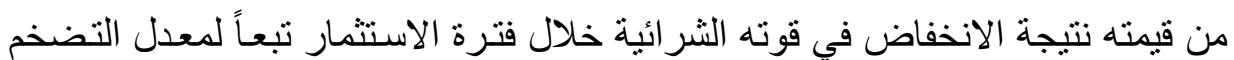

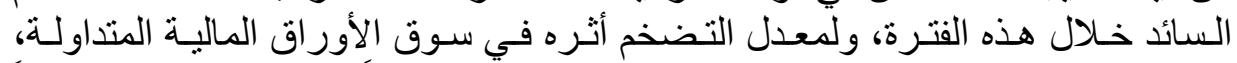

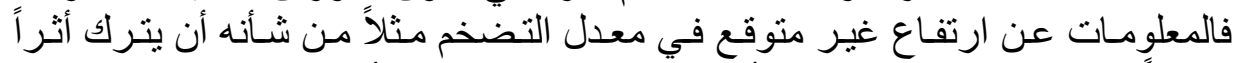

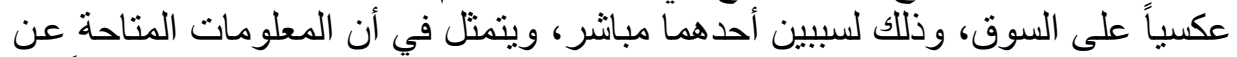

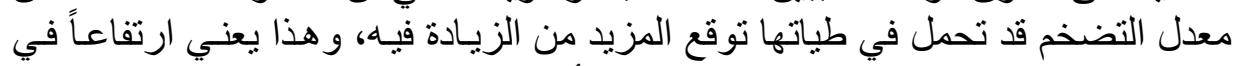

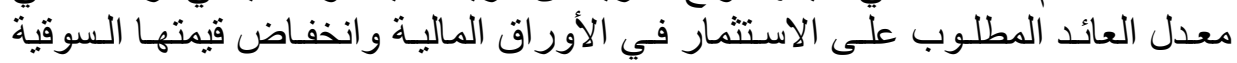

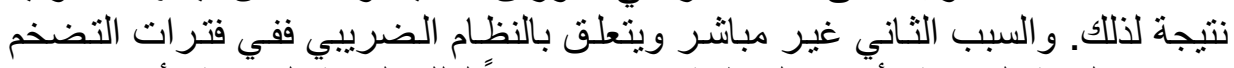

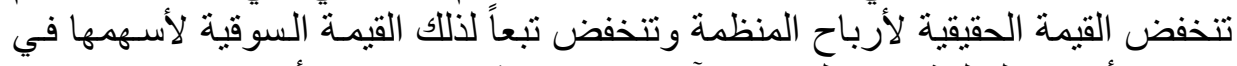

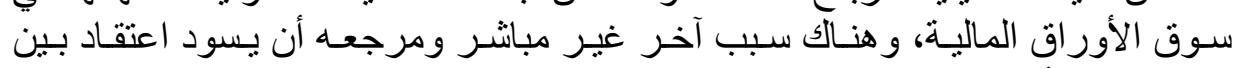

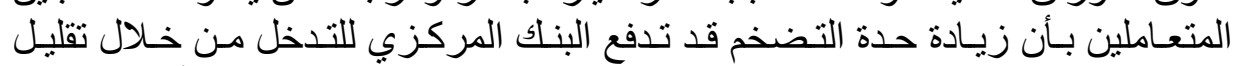

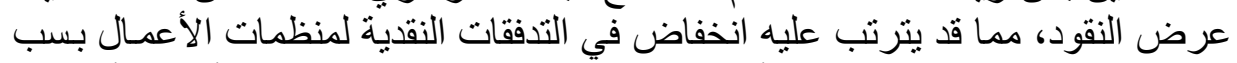

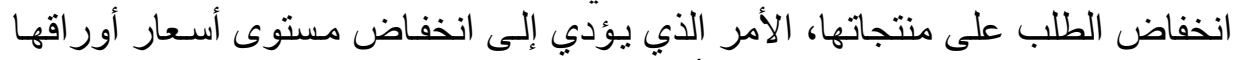

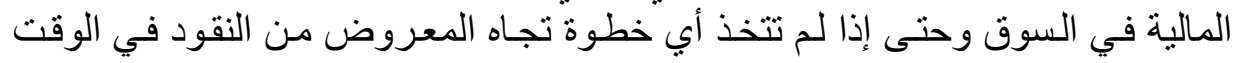

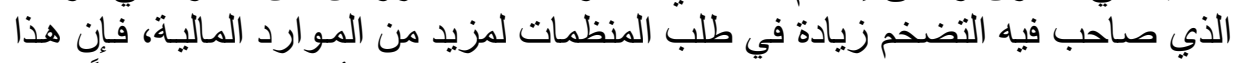

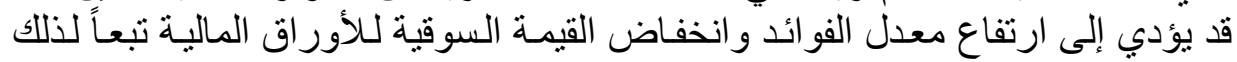

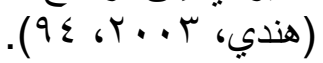

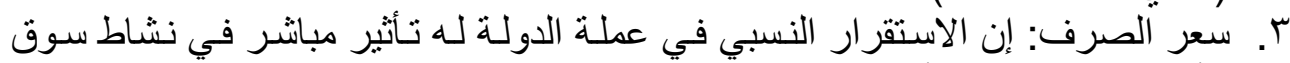

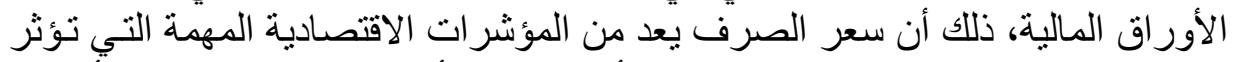

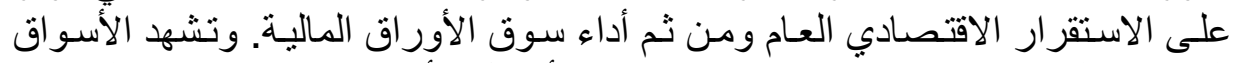

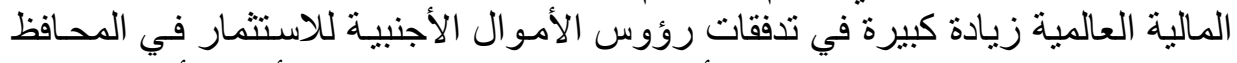

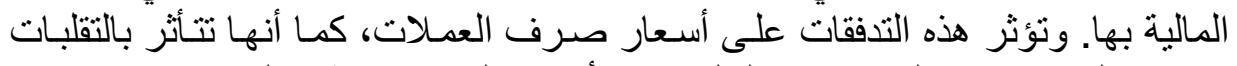

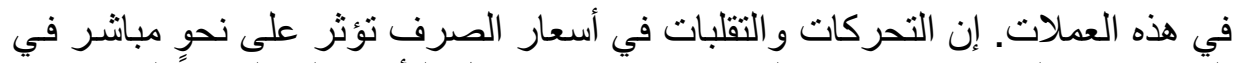

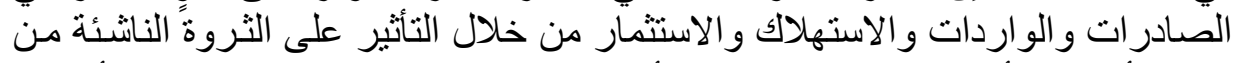

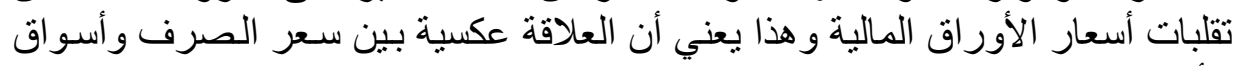

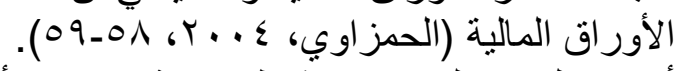

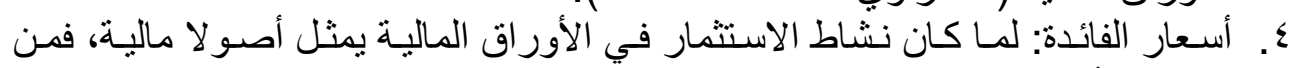

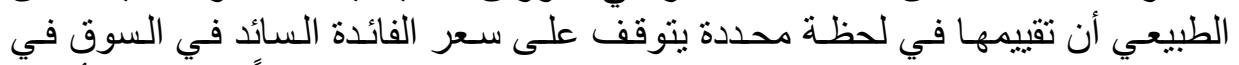

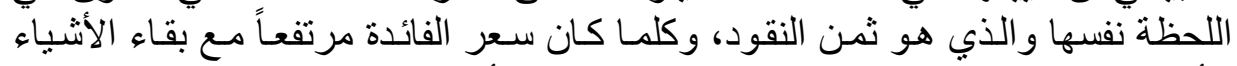

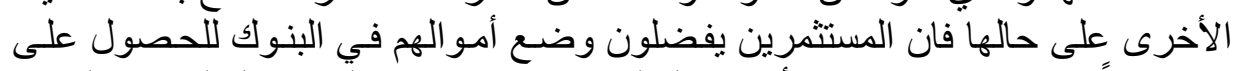

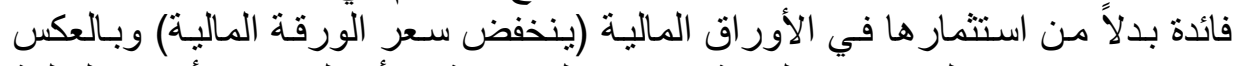

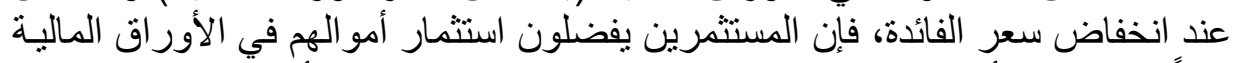

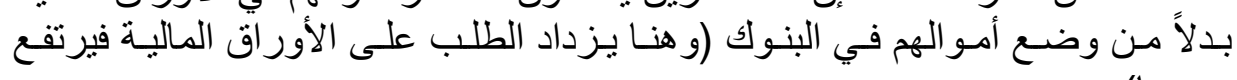

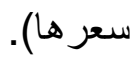
ه. السياسة الماليـة: يقصد بها وسائل تمويلـل واتجاهـات الإنفاق الحكومي وأن متغيرات

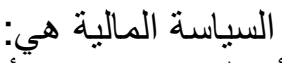
أ. الضرائب: تعد أحيد مصادر الإير ادات الرئيسة في غالبيـة الدول واللازمـة لتمويل

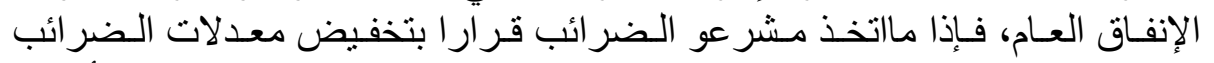

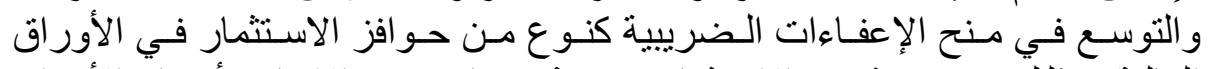

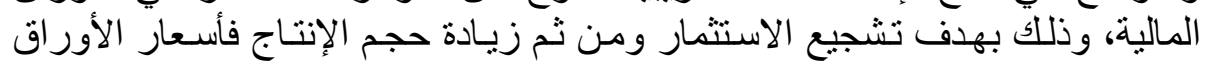




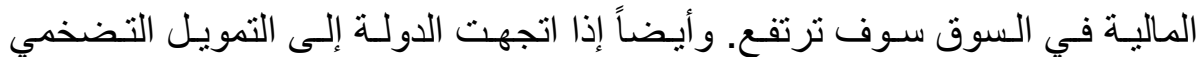

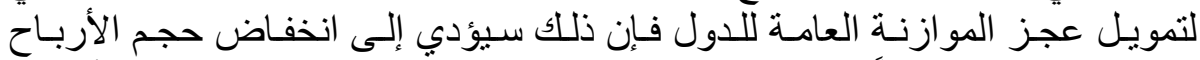

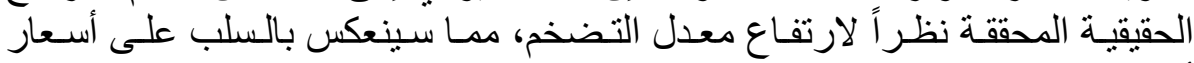

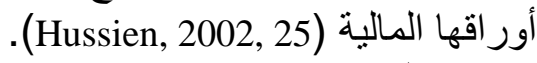

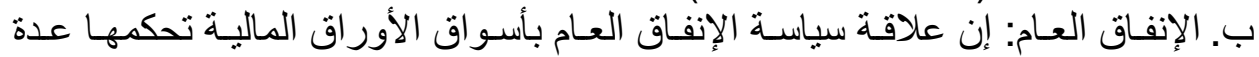

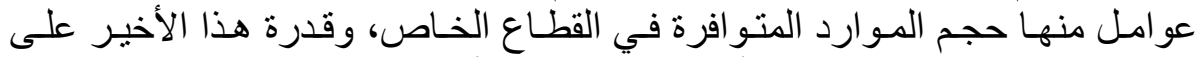

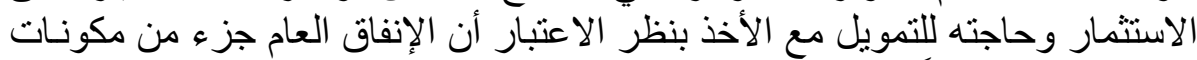

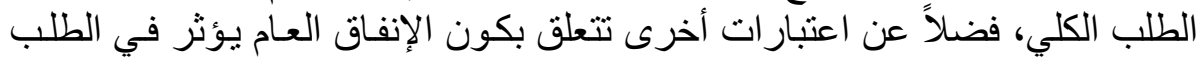

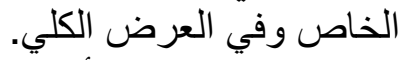

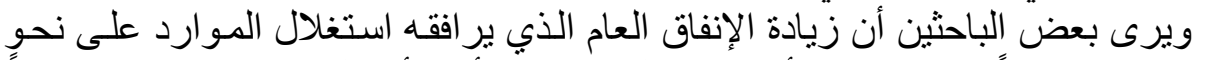

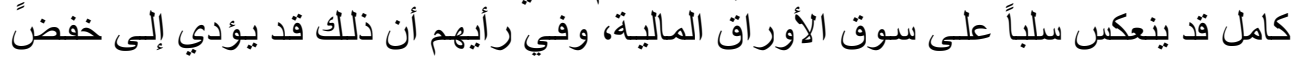

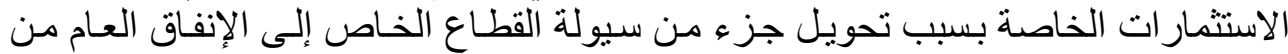

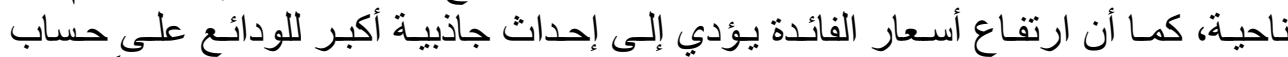

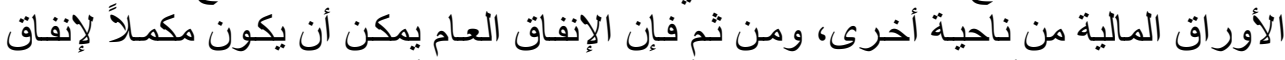

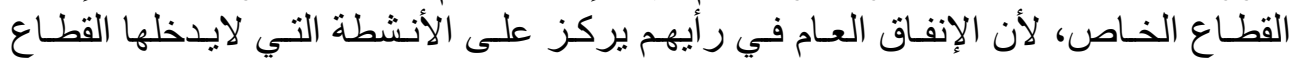

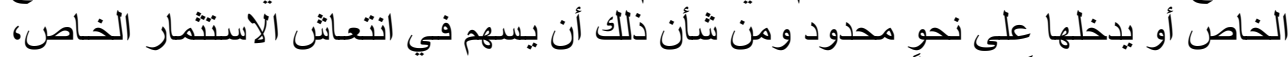
ومن ثم يؤثر إيجاباً على سوق الأوراق المالية (Mohtadi and Agarwal, 2008, 10).

ثانيـاً الدراسـات التجريبيـة السـابقة حـول النشاطات الاسـتثمارية في الأوراق الماليـة و وعلاقتها بالنمو الاقتصادي

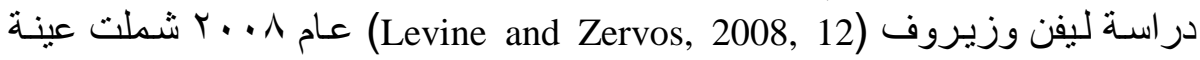

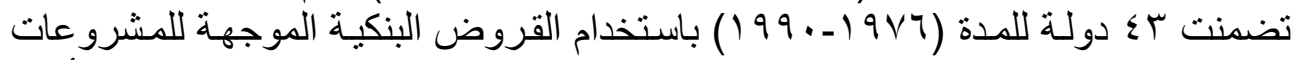

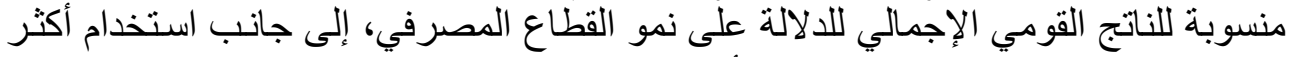

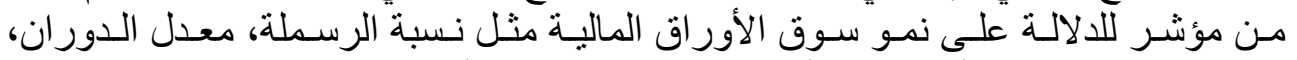

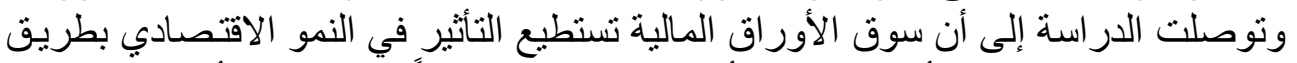

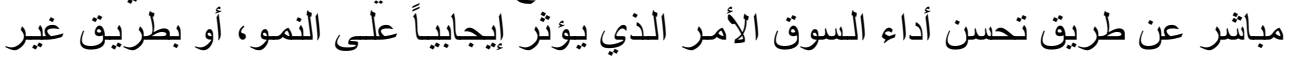

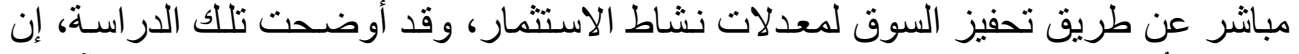

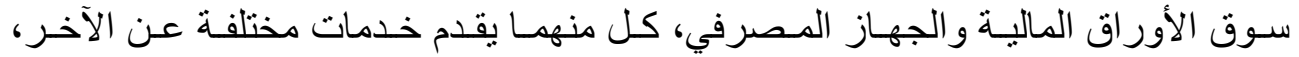
ويستطيع كل منهما التنبؤ بمعدلات النوان النمو المستقبلية.

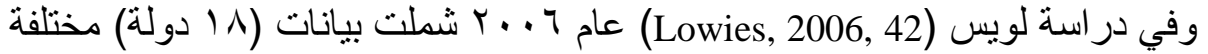

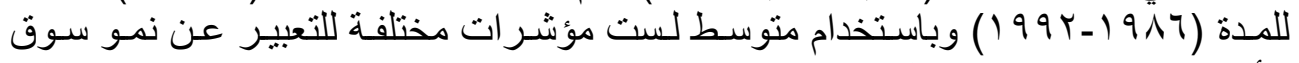

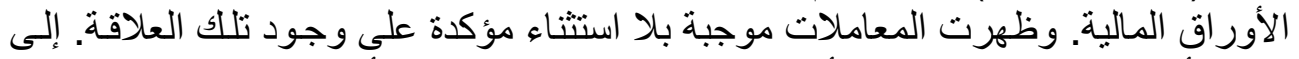

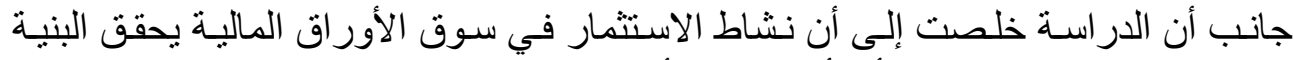

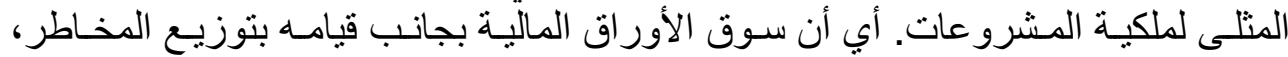

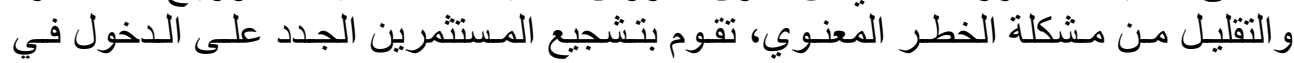

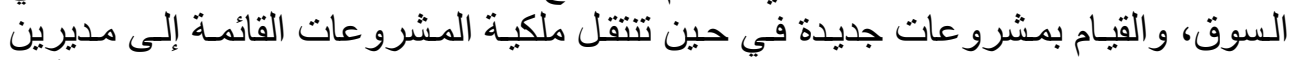

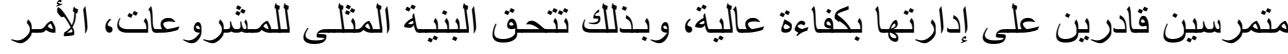

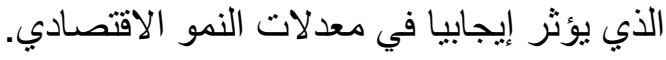




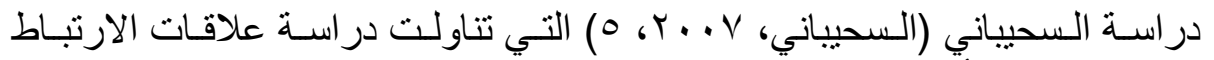

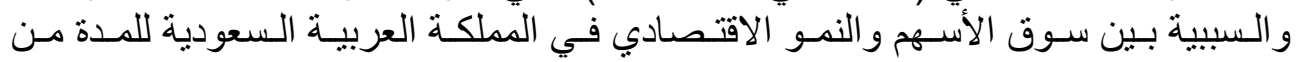

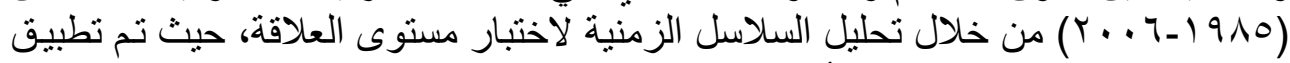

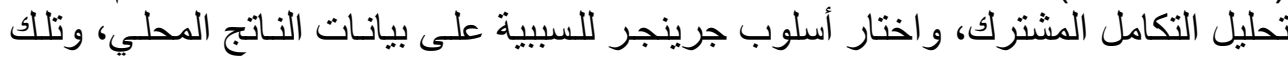

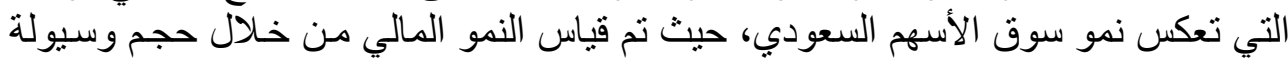

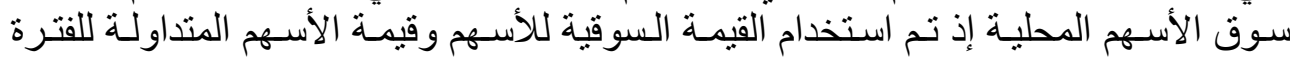

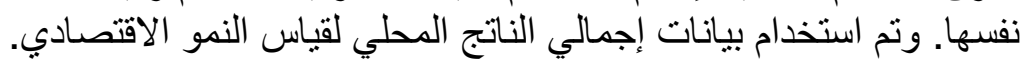

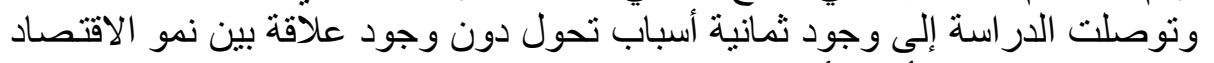

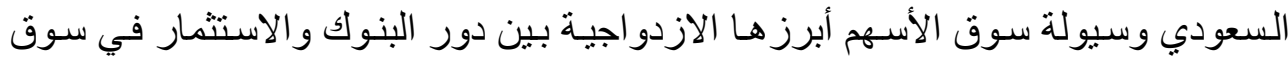

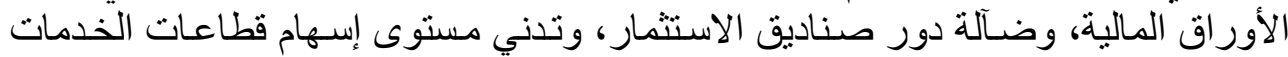

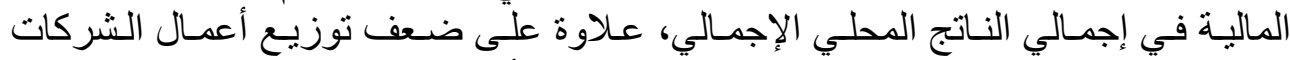

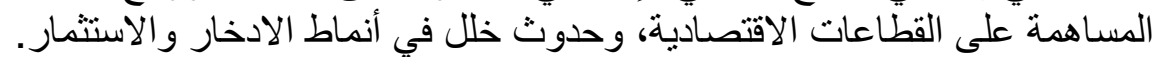

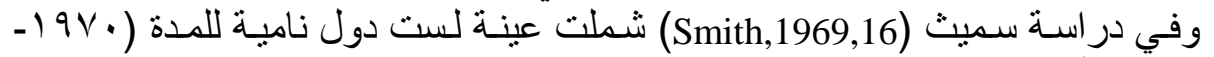

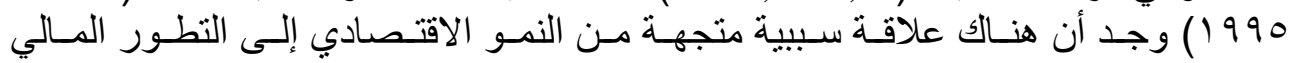

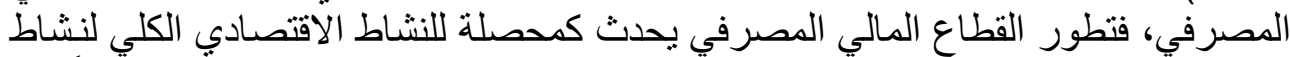

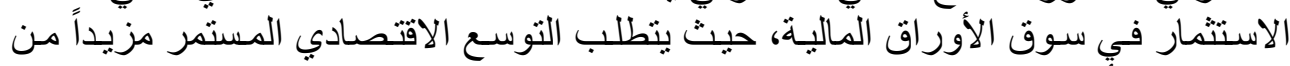

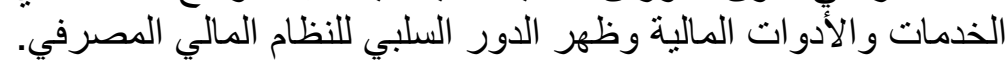

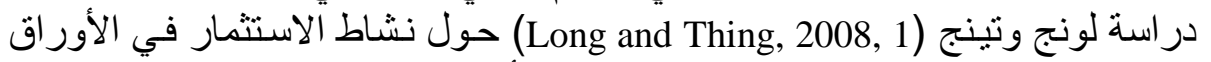

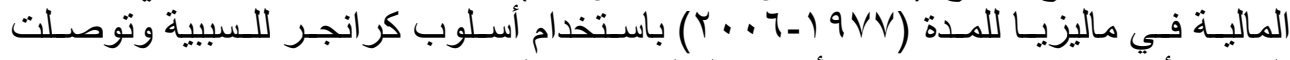

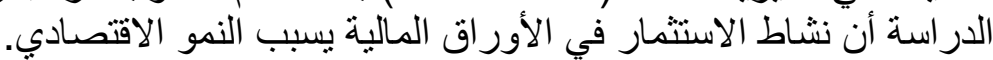

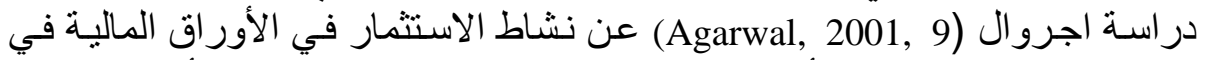

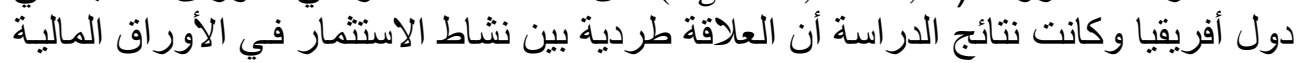

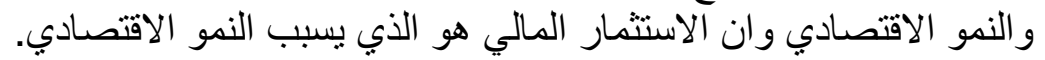

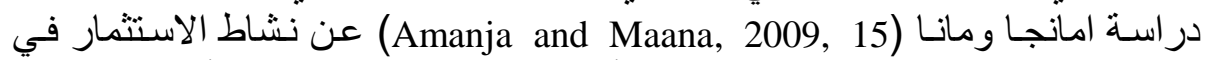

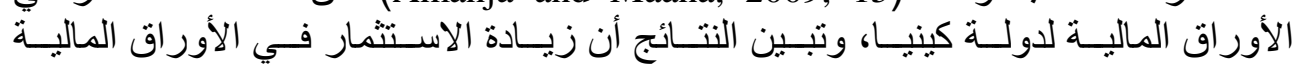

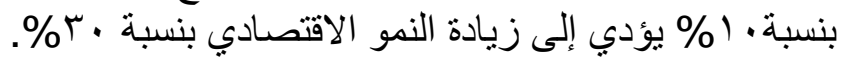

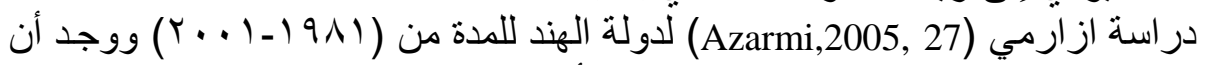

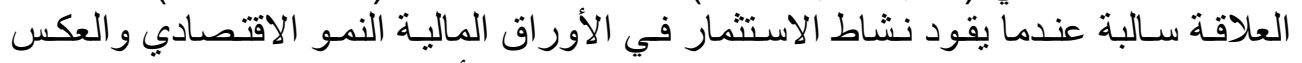

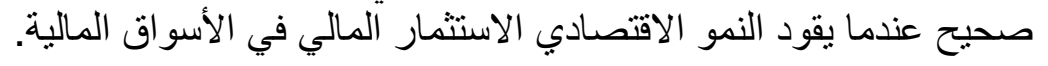

ثالثاً توصيف الأنموذج القياسي المستخدم لتحديد العلاقة بين تطور سوق الأوراق المالية

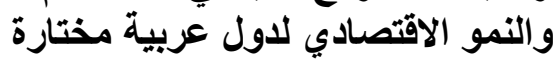

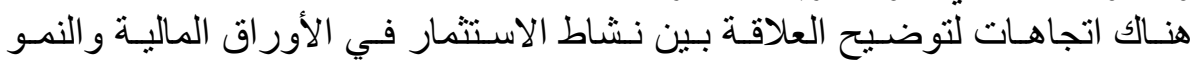

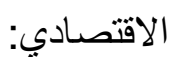
الأول: إن نشاط الاستثمار في الأوراق الماليـة ومتغيرات اقتصادية أخرى تؤُثر في

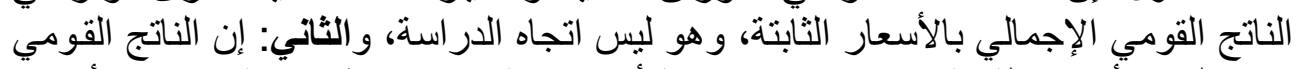

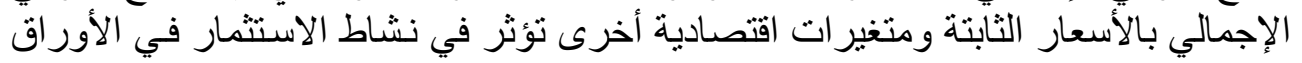

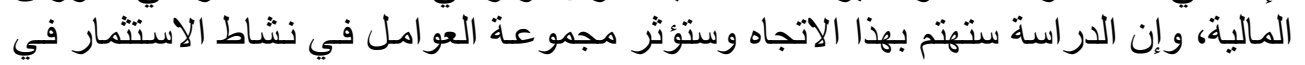


الأوراق المالية، وهي متمثلة بدالة نشاط الاستثمار في الأوراق المالية لدول عربيـة مختارة

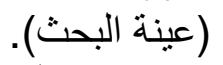

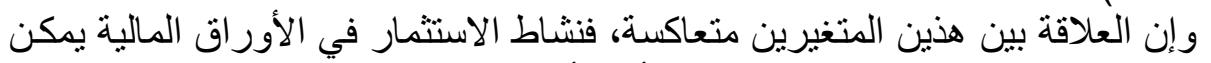

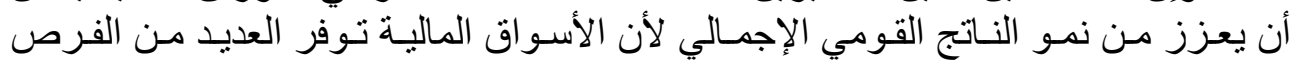

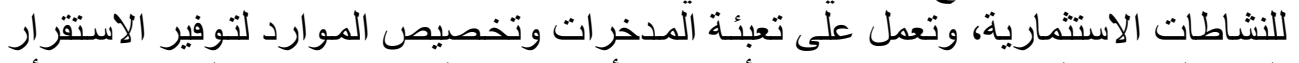

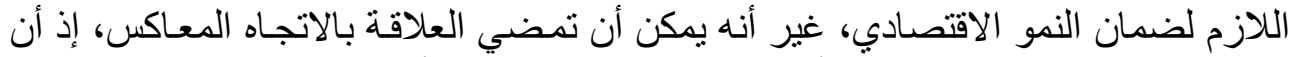

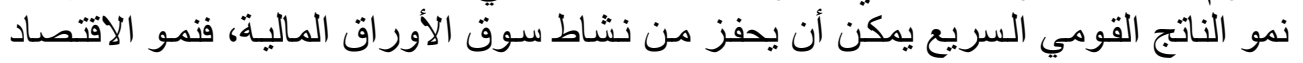

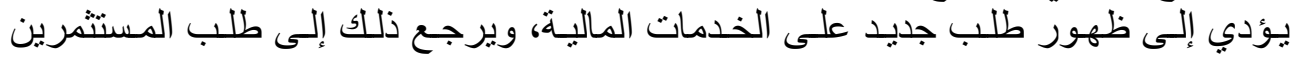
و المدخرين في القطاع الحقيقي على هذه الخدمات الخدات. كما أن العلافة بين نشاط الاستثمار في الأوراق المالية ولئه ونمو الناتج يعتمد على عو امل عديدة اقتصادية، وسياسية ونقافينة التعاطية.

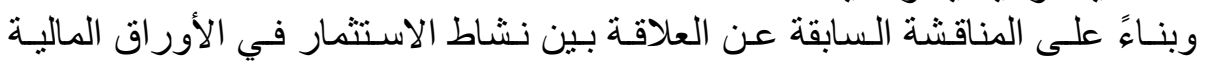

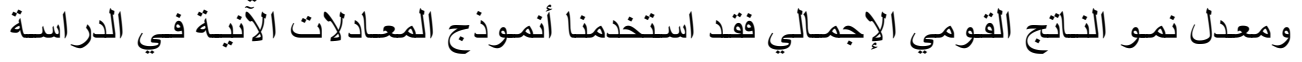
بسبب: إنب: بعض النماذج تتضمن استخدام أكثر من معادلة واحدة أي استخدام نمـاذج المعادلات الآنية. r. إن متغير ات هذه المعادلة تحقق شروط جميع المعادلات الآنيـة وتنطبق على أنموذج المعادلات الآنية.

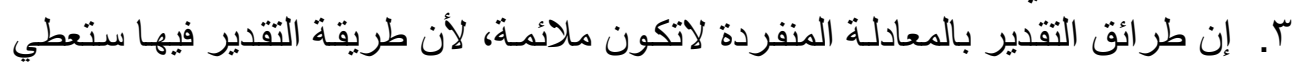

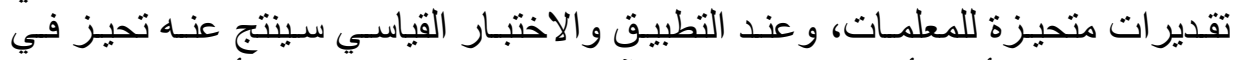

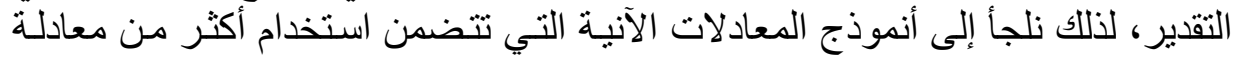

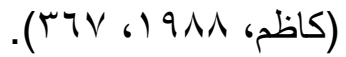

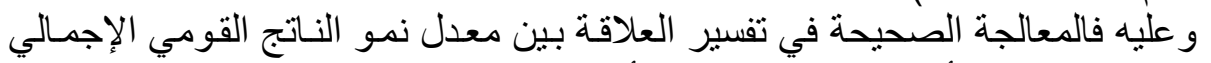

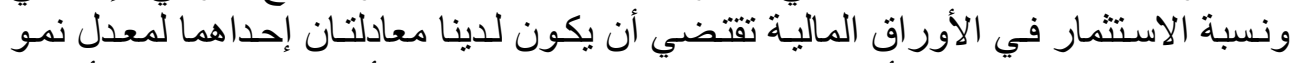

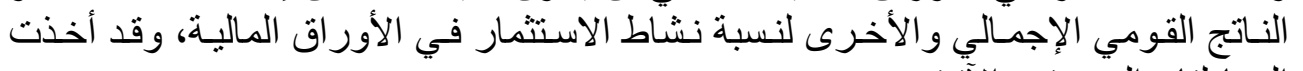
المعادلتان الصيغتين الآتيتين:

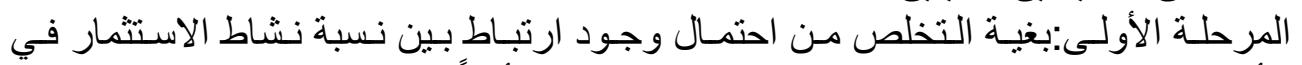

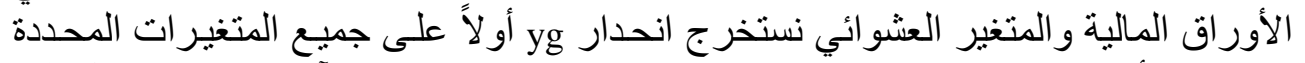

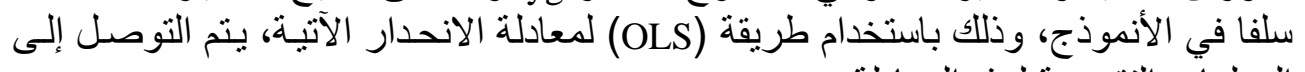
المعلمات التقديرية لهذه المعادلة:

\section{أولاً - معادلة معدل نمو الناتج القومي الإجمالي(yg)}

$\mathrm{Yg}=\mathrm{S} / \mathrm{GNP}+\mathrm{I}+\mathrm{Ex} / \mathrm{GNP}+\mathrm{p}$ 


$$
\text { EX/GNP }
$$

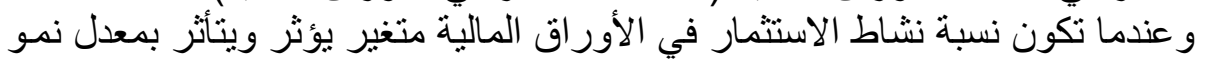

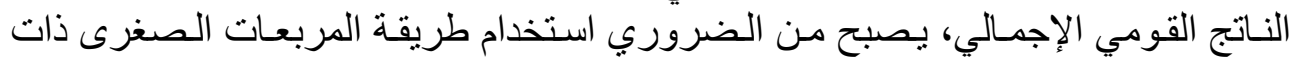

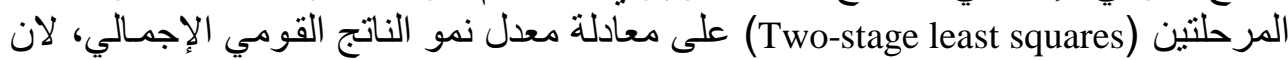

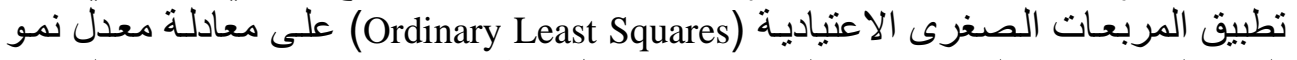

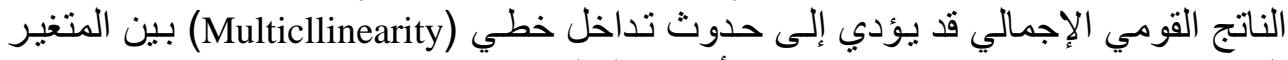

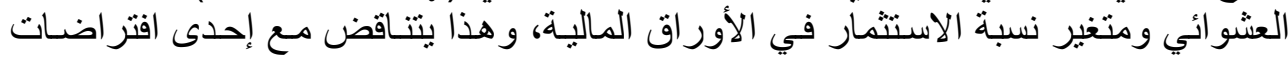

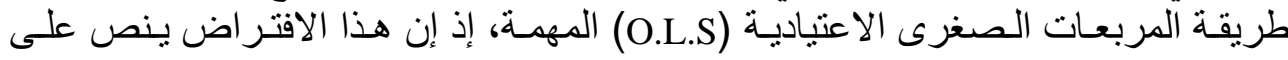

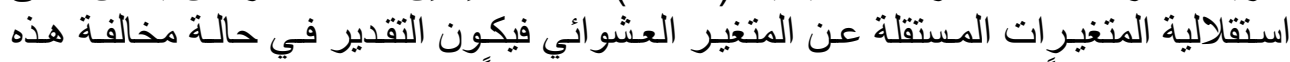

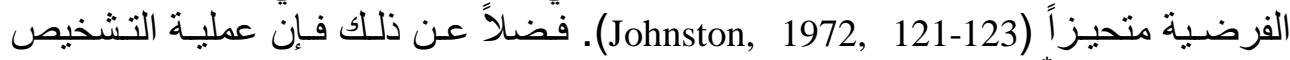
(2.S.L.S) فليها. (Identification)

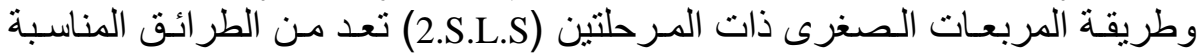

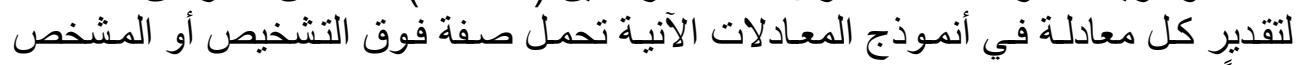

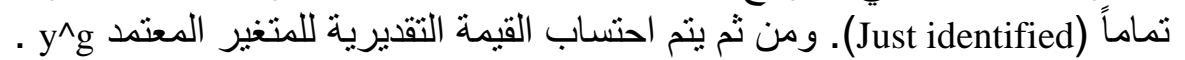

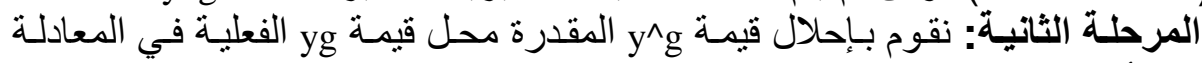

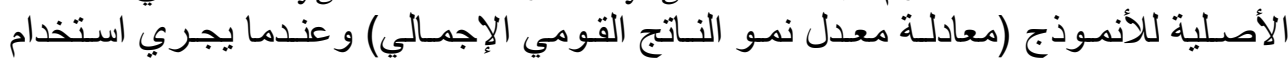

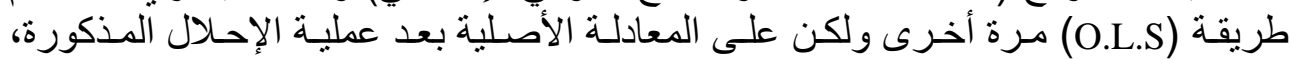

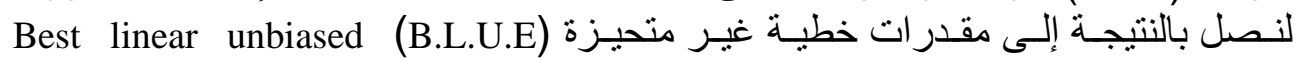
Estimators (P) ثاتياً- معادلة نشاط الأسواق في الأوراق المعادلة المالية:

$\mathrm{P}=\mathrm{GNP}+\mathrm{GG} / \mathrm{GNP}+\mathrm{I}+\mathrm{i}+\mathrm{EXG}+\mathrm{yg}^{\wedge}+\mathrm{T} / \mathrm{GNP}$

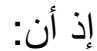
P : الاستثمار في محافظ الأوراق المالية (نشاط الاستثمار في الأوراق المالية) GNP GG/GNP I/GNP ( معدل سعر الفائدة الحقيقي : : i EXG Yg^

$\mathrm{Yg}=\mathrm{S} / \mathrm{GNP}+\mathrm{I}+\mathrm{Ex} / \mathrm{GNP}+\mathrm{P}$ T:Y/Y: الضر ائب كنسبة من الناتج القومي الإجمالي $\mathrm{P}=\mathrm{GNP}+\mathrm{GG} / \mathrm{GNP}+\mathrm{I}+\mathrm{i}+\mathrm{EXG}+\mathrm{yg}^{\wedge}+\mathrm{T} / \mathrm{GNP}$ .2

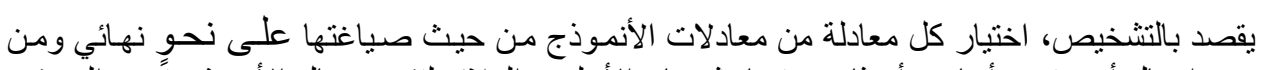

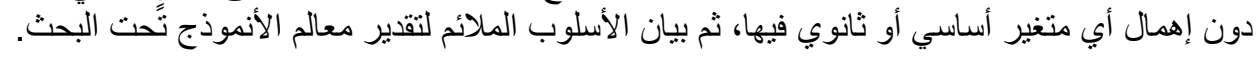




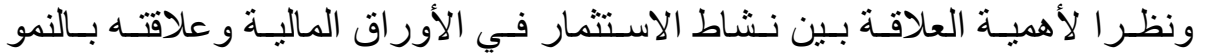

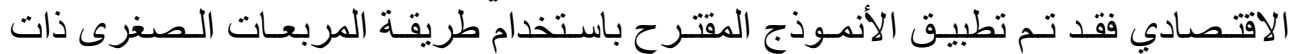

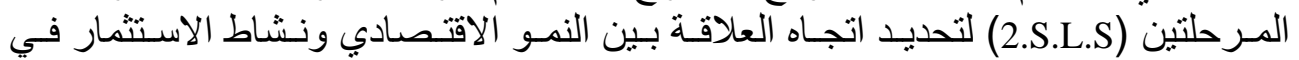

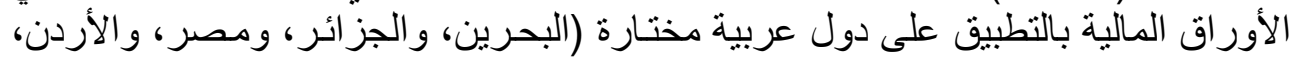

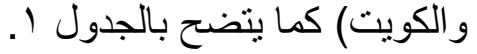

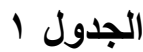

اتجاه العلاقة بين نشاط الاستثمار في الأوراق المالية والنمو الاقتصادي باستخدام

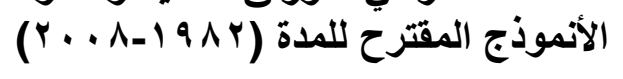

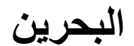

\begin{tabular}{|c|c|c|c|c|c|c|c|c|c|}
\hline $\mathbf{y}$ & bo & EX/GNP & GNP & S/GNP & inflation & Profile & \multirow[b]{2}{*}{$F=6.3$} & \multirow[b]{2}{*}{$\begin{array}{c}\mathbf{R}_{\%}^{2=28.0} \\
\end{array}$} & \\
\hline & $\begin{array}{c}7.9 \\
0.71\end{array}$ & $\begin{array}{c}0.033 \\
0.28\end{array}$ & $\begin{array}{l}0.135 \\
-0.72\end{array}$ & $\begin{array}{l}0.201 \\
-1.92\end{array}$ & $\begin{array}{l}0.106 \\
-1.22\end{array}$ & $\begin{array}{c}0.010 \\
2.06\end{array}$ & & & \\
\hline profile & bo & GNP & $\begin{array}{c}\text { Expendure/G } \\
\text { NP }\end{array}$ & $\begin{array}{c}\text { Inflation } \\
\text { rate }\end{array}$ & $\begin{array}{l}\text { Interest } \\
\text { rate }\end{array}$ & $\begin{array}{l}\text { Exchange } \\
\text { rate }\end{array}$ & $\mathrm{Y}^{\wedge}$ & T/GNP & \multirow[b]{2}{*}{$\begin{array}{l}\mathrm{F}=54.59 \\
\mathrm{R}^{2=99.1 \%}\end{array}$} \\
\hline & $\begin{array}{l}3.70 \\
1.14\end{array}$ & $\begin{array}{c}0.0077 \\
1.17\end{array}$ & $\begin{array}{l}0.222 \\
-1.76\end{array}$ & $\begin{array}{c}0.0341 \\
0.99\end{array}$ & $\begin{array}{c}0.0326 \\
0.95\end{array}$ & $\begin{array}{c}0.0005 \\
-0.69\end{array}$ & $\begin{array}{c}0.069 \\
0.42\end{array}$ & $\begin{array}{r}1.49 \\
-9.35\end{array}$ & \\
\hline
\end{tabular}

الجزائر

\begin{tabular}{|c|c|c|c|c|c|c|c|c|c|}
\hline \multirow[t]{2}{*}{$\mathbf{y}$} & bo & EX/GNP & GNP & S/GNP & inflation & Profile & \multirow[b]{2}{*}{$F=16.1$} & \multirow[b]{2}{*}{$\begin{array}{c}\mathbf{R}_{\%}^{2=89.6} \\
\end{array}$} & \\
\hline & 2.41 & $\begin{array}{c}0.0381 \\
-5.40\end{array}$ & $\begin{array}{c}0.883 \\
6.60\end{array}$ & $\begin{array}{c}0.000532 \\
2.42\end{array}$ & $\begin{array}{c}0.00039 \\
-0.64\end{array}$ & $\begin{array}{c}0.00227 \\
-0.27\end{array}$ & & & \\
\hline \multirow[t]{2}{*}{ profile } & bo & GNP & $\begin{array}{c}\text { Expendure/G } \\
\text { NP } \\
\end{array}$ & $\begin{array}{l}\text { Inflation } \\
\text { rate }\end{array}$ & $\begin{array}{c}\text { Interest } \\
\text { rate }\end{array}$ & $\begin{array}{l}\text { Exchange } \\
\text { rate }\end{array}$ & $\mathrm{Y}^{\wedge}$ & T/GNP & $\mathrm{F}=57.23$ \\
\hline & $\begin{array}{l}3.70 \\
1.04\end{array}$ & $\begin{array}{l}0.0077 \\
-2.62\end{array}$ & $\begin{array}{l}0.222 \\
-1.11\end{array}$ & $\begin{array}{c}0.0341 \\
-0.62\end{array}$ & $\begin{array}{c}0.0326 \\
-0.47\end{array}$ & $\begin{array}{c}0.0005 \\
-0.56\end{array}$ & $\begin{array}{c}0.069 \\
1.91\end{array}$ & $\begin{array}{l}1.49 \\
3.19\end{array}$ & $R^{2=70.1 \%}$ \\
\hline
\end{tabular}

\begin{tabular}{|c|c|c|c|c|c|c|c|c|c|}
\hline $\mathbf{y}$ & bo & EX/GNP & GNP & S/GNP & inflation & Profile & \multirow[b]{2}{*}{$F=4.07$} & \multirow[b]{2}{*}{$\mathbf{R}^{2=49.2 \%}$} & \\
\hline & $\begin{array}{l}0.88 \\
0.37\end{array}$ & $\begin{array}{l}0.118 \\
0.28\end{array}$ & $\begin{array}{l}0.368 \\
-0.72\end{array}$ & $\begin{array}{l}0.665 \\
-1.92\end{array}$ & $\begin{array}{l}0.0162 \\
-1.22\end{array}$ & $\begin{array}{l}0.00224 \\
2.06\end{array}$ & & & \\
\hline \multirow[t]{2}{*}{ profile } & bo & GDP & $\begin{array}{c}\text { Expendure/ } \\
\text { GNP }\end{array}$ & $\begin{array}{l}\text { Inflation } \\
\text { rate }\end{array}$ & $\begin{array}{l}\text { Interest } \\
\text { rate }\end{array}$ & $\begin{array}{c}\text { Exchange } \\
\text { rate }\end{array}$ & $\mathrm{Y}^{\wedge}$ & T/GNP & \multirow[b]{2}{*}{$\begin{aligned} F & =20.39 \\
R^{2} & =46.8 \%\end{aligned}$} \\
\hline & $\begin{array}{l}1618 \\
1.14\end{array}$ & $\begin{array}{l}4.6 \\
1.17\end{array}$ & $\begin{array}{c}30.1 \\
-1.76\end{array}$ & $\begin{array}{l}5.36 \\
0.99\end{array}$ & $\begin{array}{l}7.05 \\
0.95\end{array}$ & $\begin{array}{c}43.1 \\
-0.69\end{array}$ & $\begin{array}{c}6.8 \\
0.42\end{array}$ & $\begin{array}{l}1.49 \\
21.9\end{array}$ & \\
\hline
\end{tabular}

الاردن

\begin{tabular}{|c|c|c|c|c|c|c|c|c|c|}
\hline $\mathbf{y}$ & bo & EX/GNP & GNP & S/GNP & inflation & Profile & & & \\
\hline & $\begin{array}{l}2.81 \\
2.94 \\
\end{array}$ & $\begin{array}{c}0.1223 \\
2.05 \\
\end{array}$ & $\begin{array}{c}0.135 \\
2.04 \\
\end{array}$ & $\begin{array}{c}0.00316 \\
0.52\end{array}$ & $\begin{array}{l}0.138 \\
-1.52 \\
\end{array}$ & $\begin{array}{c}0.0091 \\
-0.30 \\
\end{array}$ & $F=5.53$ & $\mathbf{R}^{2=27.3 \%}$ & \\
\hline \multirow[t]{2}{*}{ profile } & bo & GNP & $\begin{array}{c}\text { Expendu } \\
\text { re/GNP }\end{array}$ & $\begin{array}{l}\text { Inflation } \\
\text { rate }\end{array}$ & $\begin{array}{c}\text { Interest } \\
\text { rate }\end{array}$ & $\begin{array}{l}\text { Exchange } \\
\text { rate }\end{array}$ & $\mathrm{Y}^{\wedge}$ & T/GNP & \multirow[b]{2}{*}{$F=27.56$} \\
\hline & 47.7 & 3.02 & 0.0009 & 3.71 & 0.73 & 0.201 & 20.6 & 0.0179 & \\
\hline
\end{tabular}




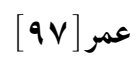

\begin{tabular}{|l|l|l|l|l|l|l|l|l|l|}
\hline & 1.22 & 1.54 & -0.04 & -2.01 & -0.55 & 1.01 & 1.81 & -0.85 & $\mathrm{R}^{2=34 \%}$ \\
\hline
\end{tabular}

الكويث

\begin{tabular}{|c|c|c|c|c|c|c|c|c|c|}
\hline $\mathbf{y}$ & bo & $\begin{array}{l}\text { EX/G } \\
\text { NP }\end{array}$ & GNP & S/GNP & inflation & Profile & \multirow[b]{2}{*}{$F=23.44$} & \multirow{2}{*}{\multicolumn{2}{|c|}{$\mathbf{R}^{2=98 \%}$}} \\
\hline & $\begin{array}{l}7.84 \\
1.33\end{array}$ & $\begin{array}{l}0.33 \\
0.03\end{array}$ & $\begin{array}{c}0.422 \\
1.02\end{array}$ & $\begin{array}{c}0.0224 \\
1.22\end{array}$ & $\begin{array}{l}0.188 \\
-2.42\end{array}$ & $\begin{array}{l}0.335 \\
1.050\end{array}$ & & & \\
\hline \multirow[t]{2}{*}{ profile } & bo & GNP & $\begin{array}{c}\text { Expendure/ } \\
\text { GNP }\end{array}$ & $\begin{array}{l}\text { Inflation } \\
\text { rate }\end{array}$ & $\begin{array}{l}\text { Interest } \\
\text { rate }\end{array}$ & $\begin{array}{l}\text { Exchange } \\
\text { rate }\end{array}$ & $\mathrm{Y}^{\wedge}$ & T/GNP & \multirow[b]{2}{*}{$\begin{array}{l}F=37.44 \\
R^{2=91 . .1 \%}\end{array}$} \\
\hline & $\begin{array}{l}61.0 \\
7.70\end{array}$ & $\begin{array}{l}0.0350 \\
-2.52\end{array}$ & $\begin{array}{r}1.16 \\
-9.92\end{array}$ & $\begin{array}{c}0.0613 \\
0.91\end{array}$ & $\begin{array}{c}0.0219 \\
-0.29\end{array}$ & $\begin{array}{c}0.461 \\
3.96\end{array}$ & $\begin{array}{c}0.602 \\
8.86\end{array}$ & $\begin{array}{l}0.602 \\
-0.86\end{array}$ & \\
\hline
\end{tabular}

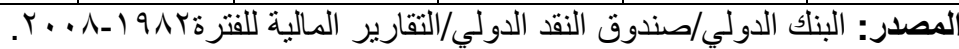

\section{نتائج التحليل والمناقشة}

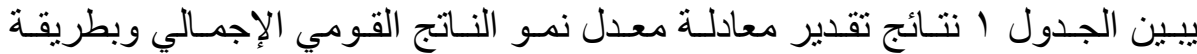

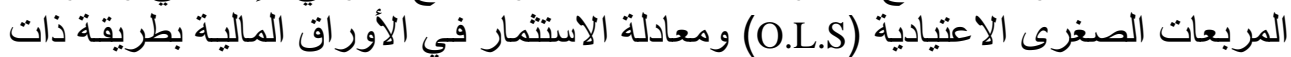

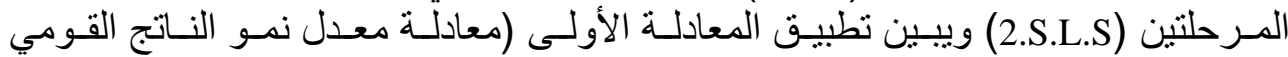

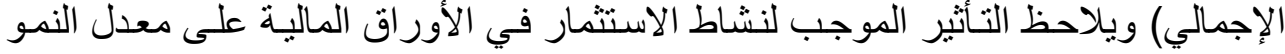

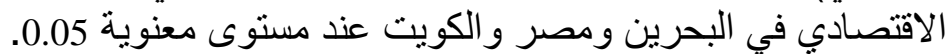

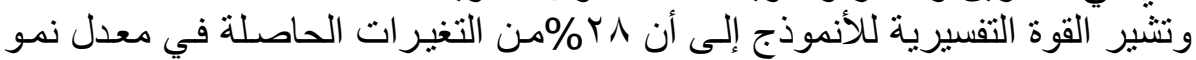

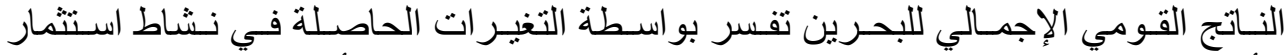

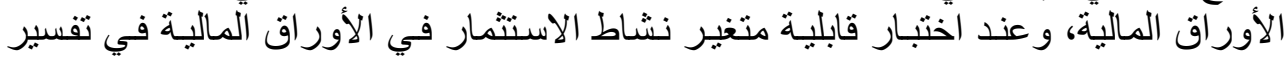

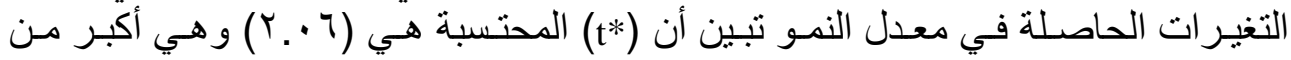

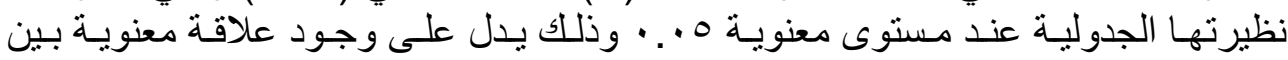

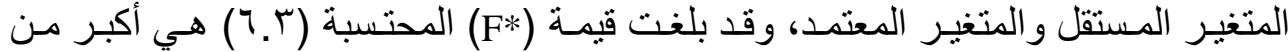

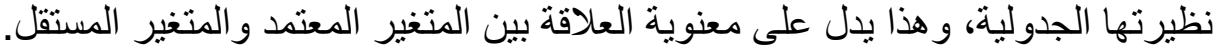

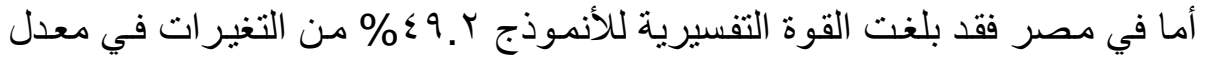

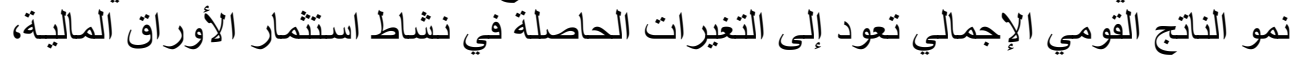

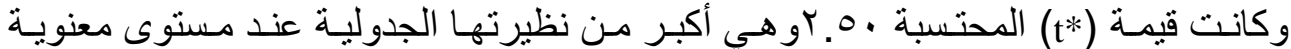

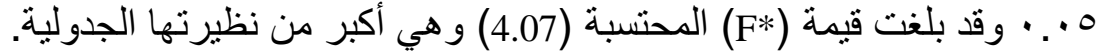

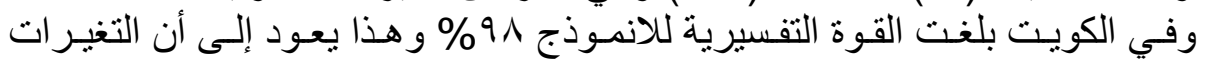

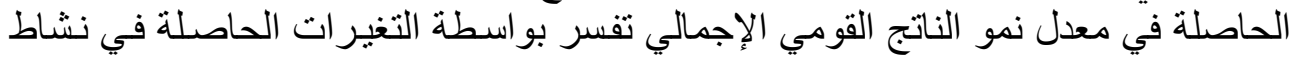

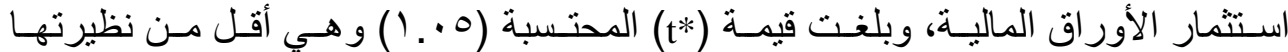
الجدولية، أما قيمة (F*) المحتسبة فقد بلغت (23.44) وهي المبرة الكبر من قيمتها الجدولية.

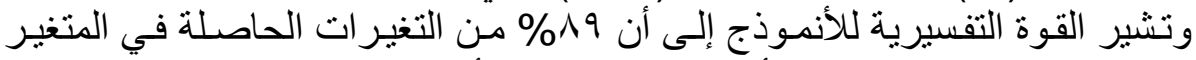

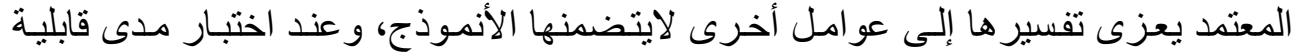

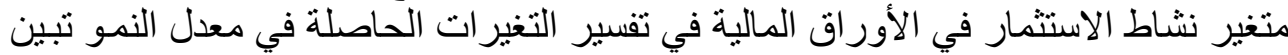

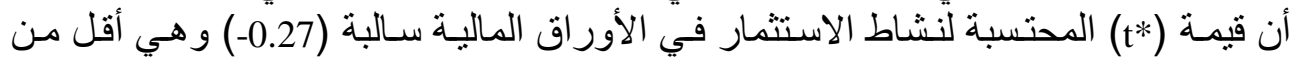


نظيرتها الجدولية، و هذا يعني عدم معنويـة العلاقـة بين المتغير المستقل و المتغير المعتمد. و اتضح بأن قيمة F* (16.1) وهي أكبر من نظيرتها الجدولية عند مستوى معنوية 0.05 .

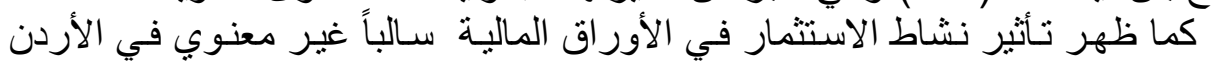
عند مستوى معنوية 0.05 .

أما بالنسبة للمعادلة الثانية معادلة نشاط الاستثمار في الأوراق المالية بتطبيق طريقة

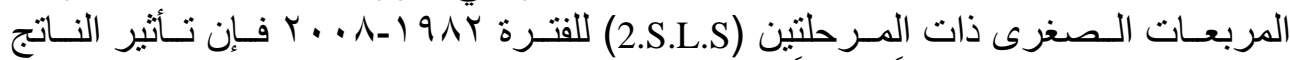

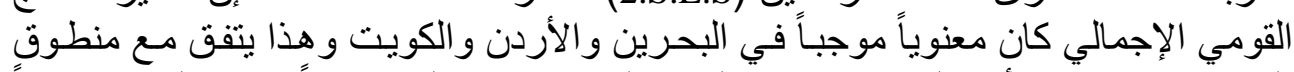

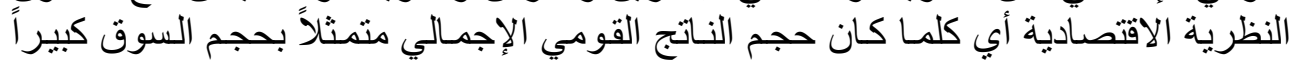

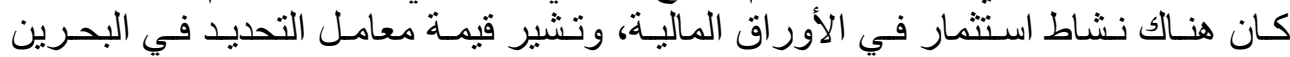

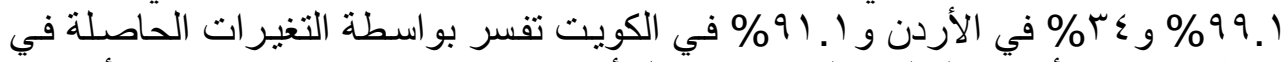

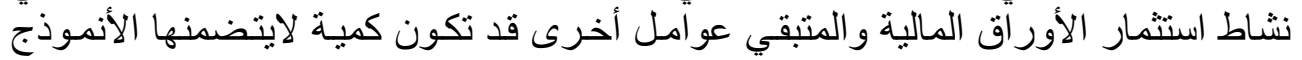

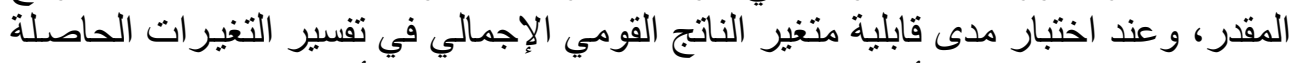

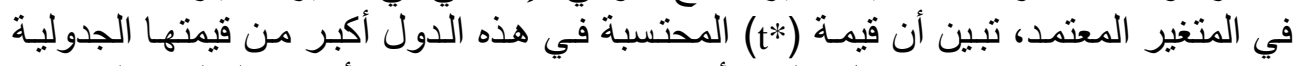

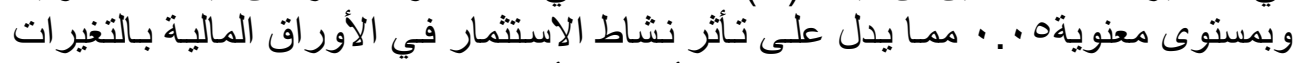

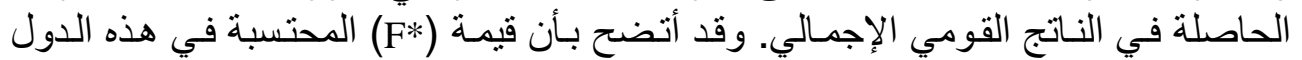

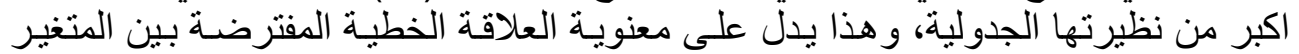

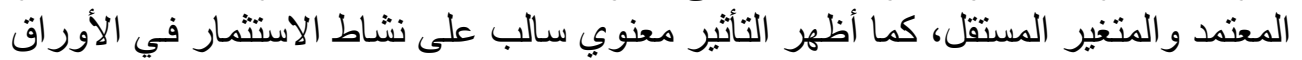
المالية في مصند والفير الجزائر.

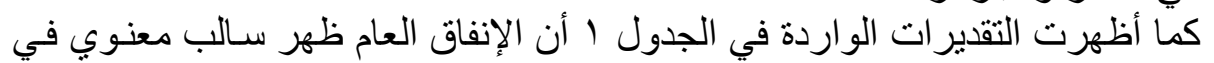

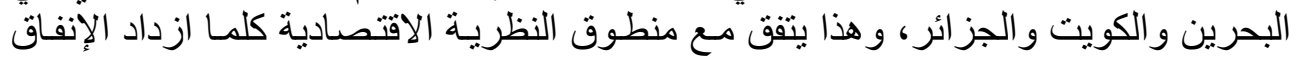

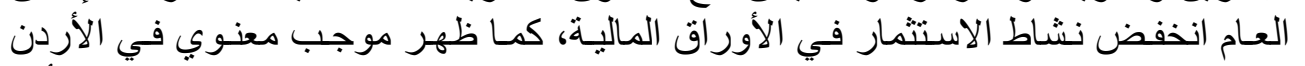

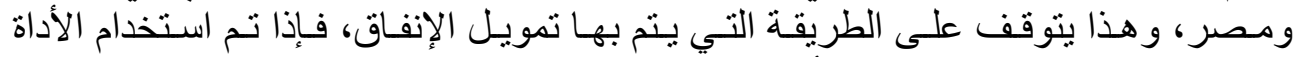

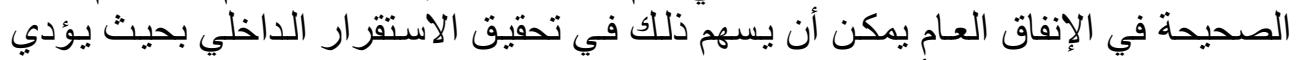

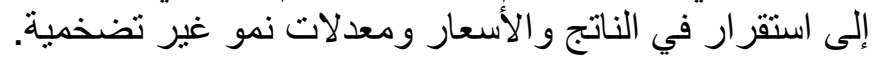

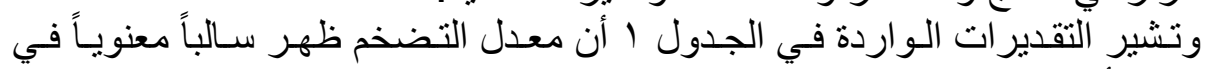

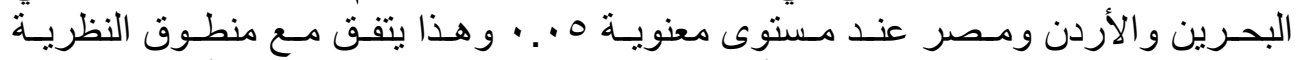

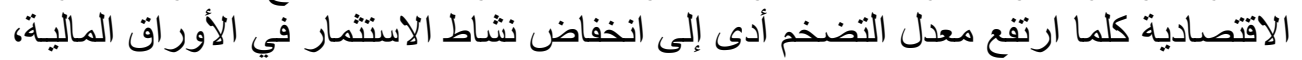

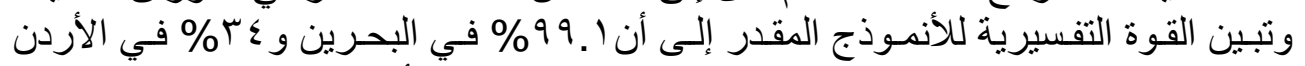

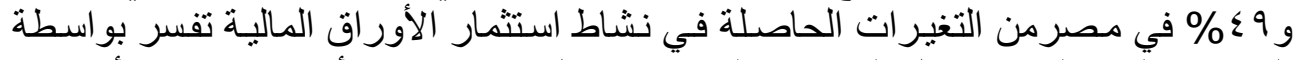

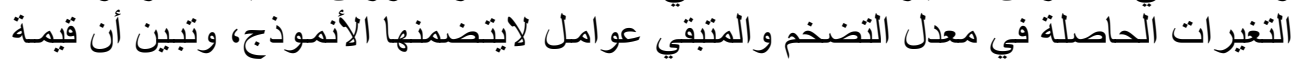
(t*)

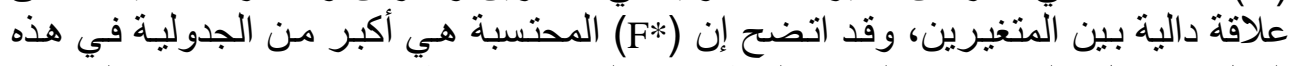

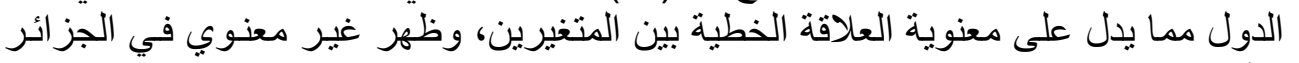

كذلك تظهر التقديرات أن تأثثر معدل سعر الفائدة قي نشاط استثمار الأوراق الماليـة و الكويت.

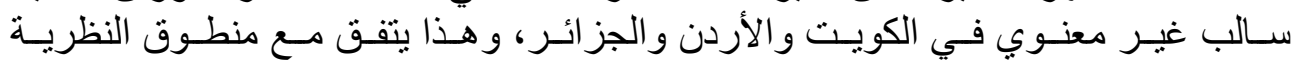

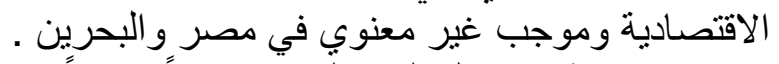

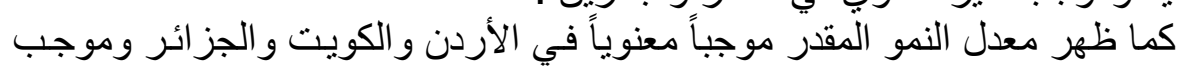
غير معنوي في مصر و البحرين. 


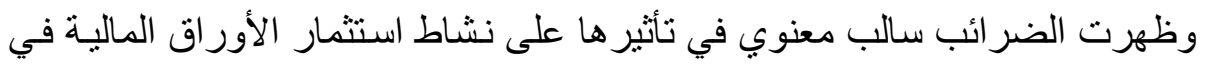

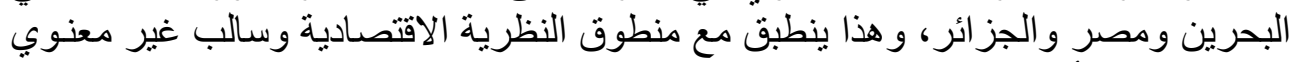

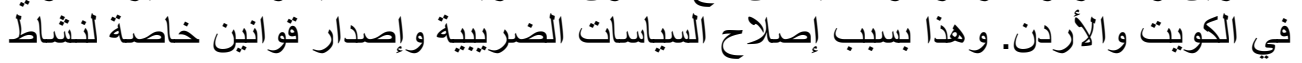

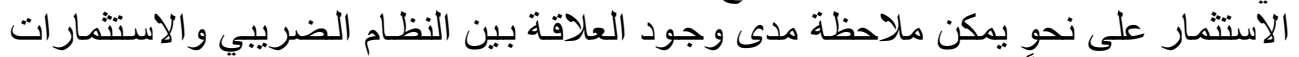

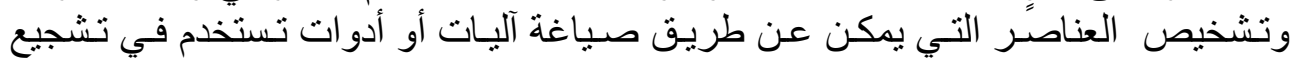
نشاط الاستثمار في الأسواق المالية.

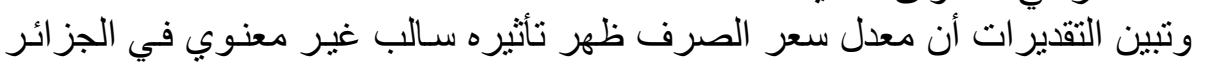

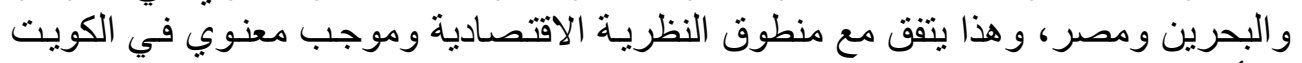

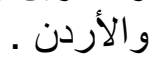

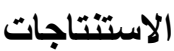

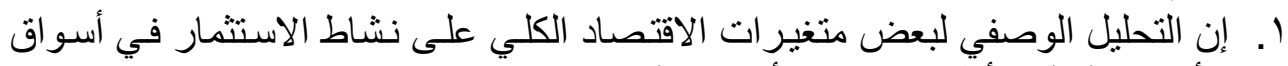

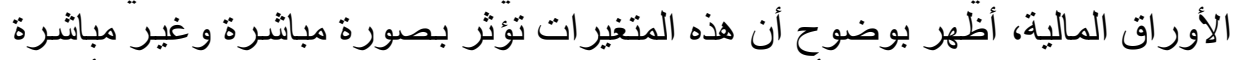

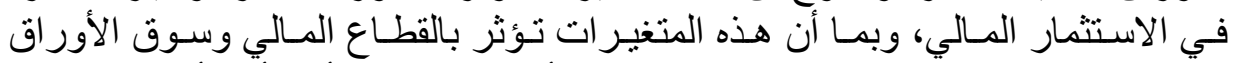

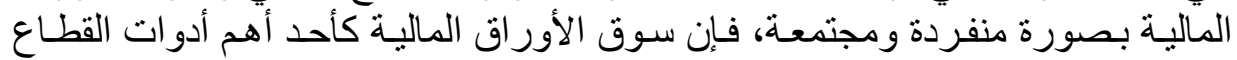

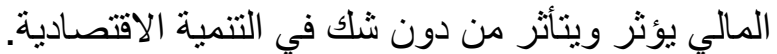

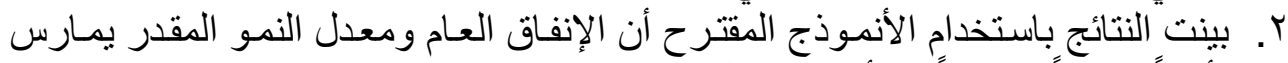

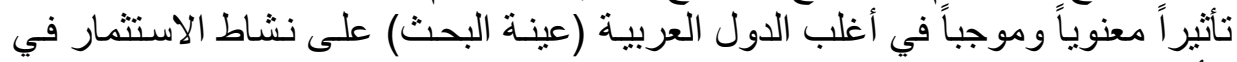

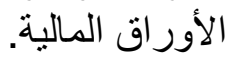

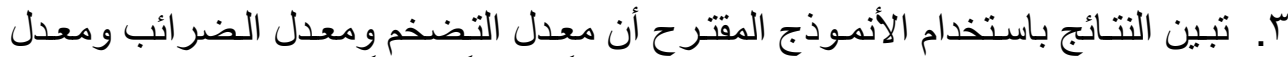

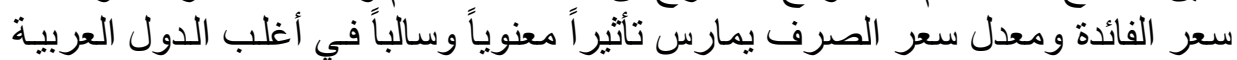
(عينة البحث). ع. تبين تطبيق الأنموذج في الدول العربية (عينة البحث) أن العلاقة بين نشاط الاستثمار المالي و النمو الاقتصادي هي علاقة ذات اتجاهين.

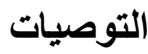

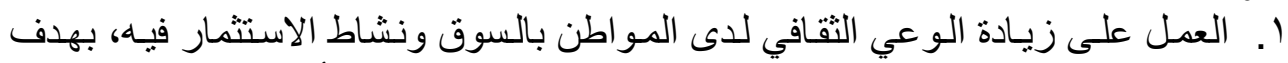

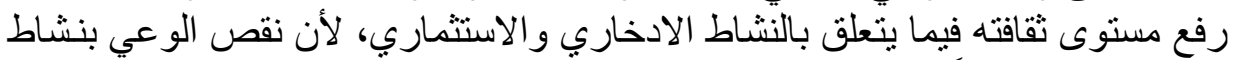

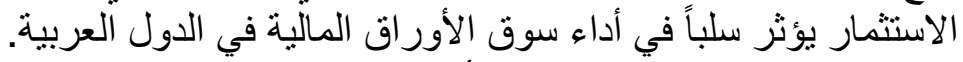

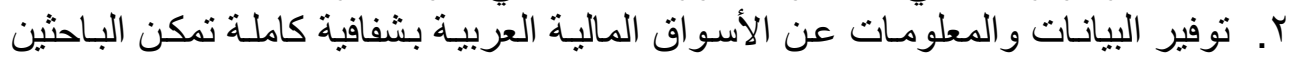

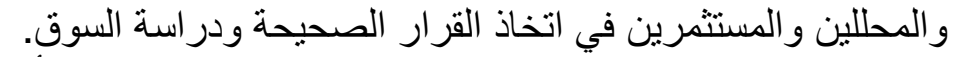

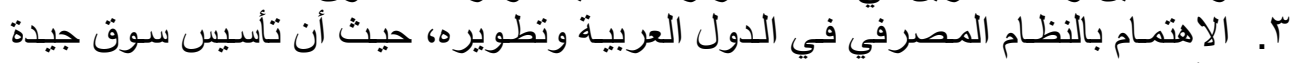

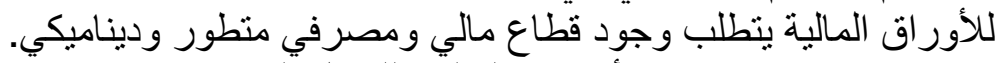

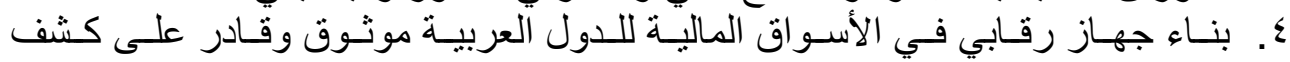

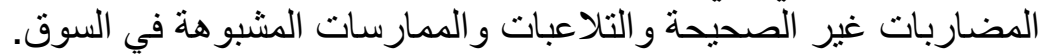

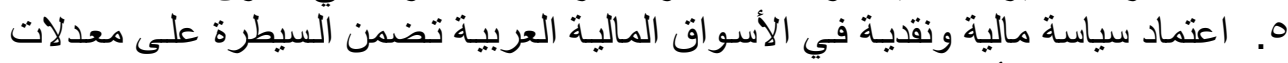
التضخم وتمنع تأثير ها على التوق ولى ولى الاقتصاد الوطني. 


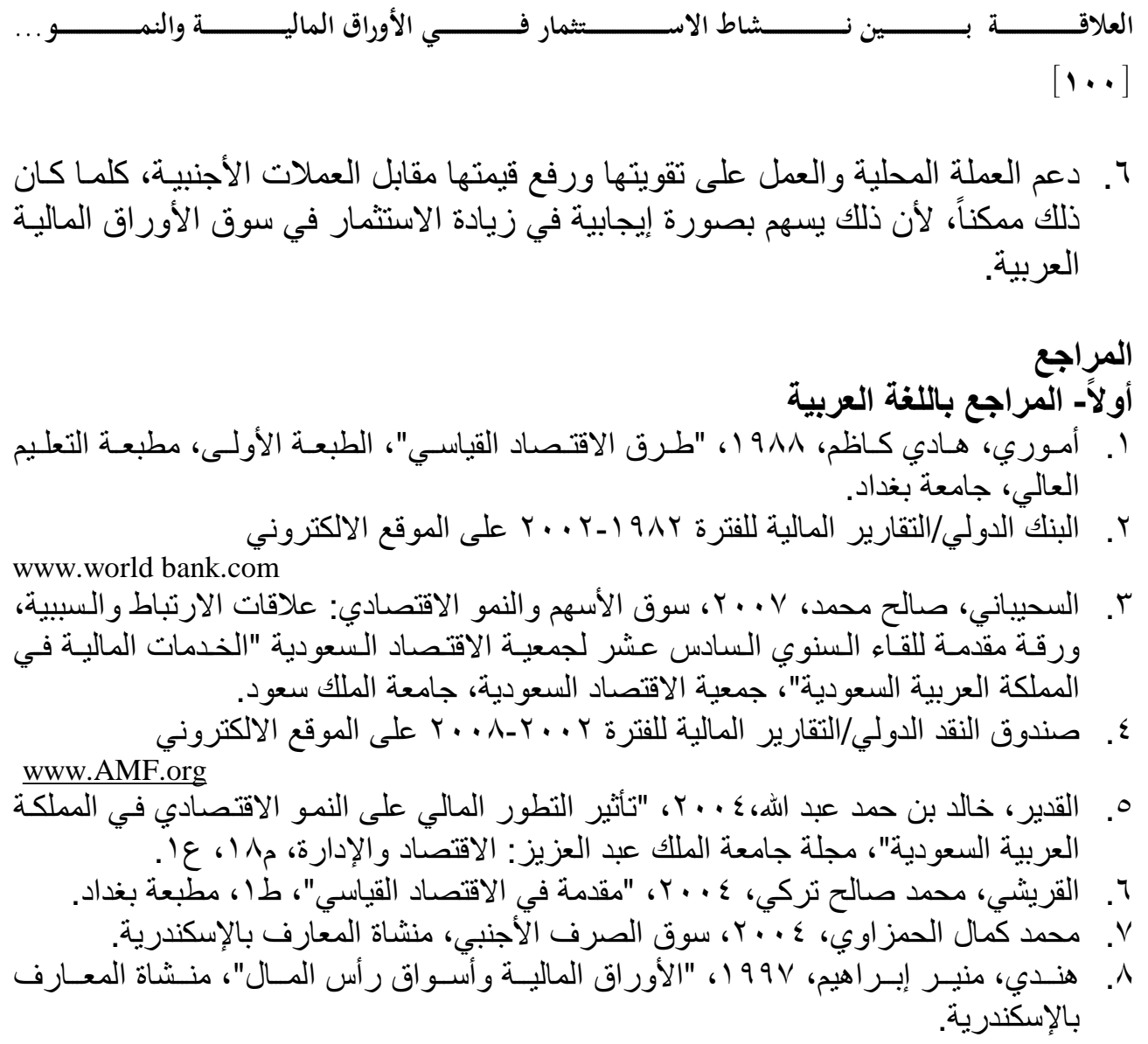

\section{ثاتياً المراجع باللغة الاجنبية}

1. Adm Lowies, The Links Between Financial Structure and Growth". ideas. www. applied.org/Issn 1546-9239.

2. Abd Almtalib Hussein, "Role of the Egyptian securities market on Saving Development" http://mpra.ub.uni-muenchen.de/2317,2002.

3. Antonio Loues, "The Role of Capital Markets in Economic Growth". ideas.repcc.org/p/rio/textdis/342.html.

4. BandarLong,B. and Tan Thing "Stock Markets and Economic Growth InMalaysia: Causality Test", ccsent.org/journal/index.php/ass/article/view file/1605/1519.2008

5. Bradford and Long "Equipment Investment and Economic Growth"citeseerx.ist.psu.edu/viewdoc/download?doi=10.1.1.165.1160,1990.

6. Gold Smith "Financial Development in African Countries" ,www.microsoft.com/pres.

7. Hamid Mohtaadi and Sumit Agarwal,"Stock Market Development and Economic Growth Evidence from Developing countries" pantherfile Uwm.edu/mohtadi/www/pA1-4-01.pdf.2008.

8. John Johnston,"Economic Methods"2 ${ }^{\text {nd }}$ Ed., McGraw-Hill Kogakusha, New York, 1972. 


\section{$[1 \cdot 1]$}

9. Rose Ngugi, Daniel Amanja and Isaya Maana "Capital Market, Financial Deeping and Economic Growth in Kenya".www.csae.ox.ac.uk /conferences/2004-EDiA/papers/513Isaya.

10. Ross Levine and Sara Zervos,"Stock Markets Banks and Economic Growth",www.jstor.org.2008.

11. Sumit Agarwal, "Stock Market Development and Economic Growth: Preliminary Evidence From African Countries ",www.jsd- africa-com/jsda/spring2001/articles pdf/ARC $\% 20.2001$

12. Sem Robert, "Profile The Economic Impact", www.microsoft.com/presspass/events/wwpc/docs/win7.

13. Ted Azarmi"Is The Indian Stock Market ACasino? www.cluteinstilute Online journals.com/pdfs/2005202.pdf.2005.

14. Thorvaldur Gylfason and Gylfi Zoega "Natural Resources and Economic Growth: The Role of Investment", ideas.repec.org/kud/epruwp/01-02.html.2002.

15. Vazakidis and Adamopoulos"A Causal Relationship between Financial Market Development and Economic Growth",www.scipub.org/fulltext/ajas/ajas74575-583.pdf. 